ARTICLE

\title{
Regulation of T-cell activation and migration by the kinase TBK1 during neuroinflammation
}

Jiayi Yu ${ }^{1, \star}$, Xiaofei Zhou ${ }^{1, \star}$, Mikyoung Chang ${ }^{1}$, Mako Nakaya ${ }^{1,2}$, Jae-Hoon Chang ${ }^{1,3}$, Yichuan Xiao ${ }^{1}$, J. William Lindsey ${ }^{4}$, Stephanie Dorta-Estremera ${ }^{1,5}$, Wei Cao ${ }^{1,5}$, Anna Zal ${ }^{1}$, Tomasz Zal 1,5,6

$\&$ Shao-Cong Sun $1,5,6$

Development of an immune or autoimmune response involves T-cell activation in lymphoid organs and subsequent migration to peripheral tissues. Here we show that T-cell-specific ablation of the kinase TBK1 promotes T-cell activation but causes retention of effector T cells in the draining lymph node in a neuroinflammatory autoimmunity model, experimental autoimmune encephalomyelitis (EAE). At older ages, the T-cell-conditional TBK1-knockout mice also spontaneously accumulate T cells with activated phenotype. TBK1 controls the activation of AKT and its downstream kinase mTORC1 by a mechanism involving TBK1-stimulated AKT ubiquitination and degradation. The deregulated AKT-mTORC1 signalling in turn contributes to enhanced T-cell activation and impaired effector T-cell egress from draining lymph nodes. Treatment of mice with a small-molecule inhibitor of TBK1 inhibits EAE induction. These results suggest a role for TBK1 in regulating T-cell migration and establish TBK1 as a regulator of the AKT-mTORC1 signalling axis.

\footnotetext{
${ }^{1}$ Department of Immunology, The University of Texas MD Anderson Cancer Center, 7455 Fannin Street, Box 902, Houston, Texas 77030, USA. ${ }^{2}$ Division of Pharmacology, School of Medicine, University of Fukui, Fukui 910-8507, Japan. ${ }^{3}$ College of Pharmacy, Yeungnam University, Gyeongsan 712-749, Republic of Korea. ${ }^{4}$ Department of Neurology, University of Texas Health Science Center at Houston, Houston, Texas 77030, USA. ${ }^{5}$ The University of Texas Graduate School of Biomedical Sciences at Houston, Houston, Texas 77030, USA. ${ }^{6}$ Center for Inflammation and Cancer, The University of Texas MD Anderson Cancer Center, 7455 Fannin Street, Box 902, Houston, Texas 77030, USA. * These authors contributed equally to this work. Correspondence and requests for materials should be addressed to S.-C.S. (email: ssun@mdanderson.org).
} 
A utoimmunity occurs as a result of T-cell activation by antigens derived from self-tissues ${ }^{1}$. Following priming by the self-antigens in peripheral lymphoid organs, autoimmune effector $\mathrm{T}$ cells migrate to target organs to mediate inflammation and tissue damage. The central nervous system (CNS) is an organ of a number of autoimmune and inflammatory disorders, including multiple sclerosis (MS), a disease characterized by chronic inflammation, demyelination and neuronal damage ${ }^{2}$. An animal model, experimental autoimmune encephalomyelitis (EAE), has proved to be powerful for investigating the pathogenesis of $\mathrm{MS}^{3}$. It is generally believed that in MS and EAE, autoimmune T cells are primed by myelin-specific antigens and then migrate across the blood-brain barrier to enter the CNS, where they become reactivated and mediate inflammation and neuronal damage ${ }^{4,5}$. The T-cell priming and differentiation are governed by signal transduction mediated by the TCR and a costimulatory molecule, CD28, as well as cytokine signals ${ }^{6}$. However, the signalling mechanism that regulates $\mathrm{T}$-cell migration from the lymphoid organs to the tissues of autoimmunity, such as CNS, is still poorly defined.

TBK1, as well as its homologous kinase IKKe, are known as mediators of type I interferon (IFN) induction in antiviral innate immunity $^{7-11}$. TBK1 and IKKe share structural homology with IKK $\alpha$ and IKK $\beta$, typical IKK components mediating activation of the transcription factor NF- $\kappa B^{12,13}$. However, unlike the typical IKKs, TBK1 and IKKe are dispensable for NF- $\kappa$ B activation but are required for activation of IFN-responsive factor 3, a transcription factor mediating type I IFN gene expression ${ }^{14}$. To date, the roles of the atypical IKKs in other biological processes are poorly defined. In particular, the study of the in vivo function of TBK1 has been hampered by the embryonic lethality of the conventional TBK1-knockout (KO) mice ${ }^{15}$.

In the present study, we employed a conditional Tbk1-KO approach and demonstrated an unexpected role for TBK1 in the regulation of T-cell function and autoimmunity. T-cell-specific ablation of TBK1 perturbed T-cell homeostasis, characterized by an increased frequency of $\mathrm{T}$ cells with an activated phenotype, and rendered naïve $\mathrm{T}$ cells more sensitive to activation by agonistic antibodies for TCR and CD28. Surprisingly, the T-cellconditional Tbk1-KO (hereafter called Tbk1-TKO) mice were refractory to the induction of EAE due to impaired migration of autoimmune $\mathrm{T}$ cells from the draining lymph nodes to the CNS. Our data suggest that TBK1 mediates egress of effector T cells from draining lymph nodes in a mechanism that involves negative regulation of the kinases AKT and mTORC1.

\section{Results}

TBK1 is a kinase that responds to T-cell activation signals. Our initial analysis of the BioGPS database revealed that in addition to macrophages, lymphocytes had an abundant expression of TBK1 (data not shown). To assess the function of TBK1 in T cells, we examined its ability to respond to signals stimulated by the TCR and CD28 agonistic antibodies (anti-CD3 and anti-CD28) or mitogens (PMA and ionomycin) that activate protein kinase $\mathrm{C}$ and calcium pathways downstream of the TCR and CD28. Despite being known as an innate immune mediator, TBK1, as well as its homologue IKKe, were strongly activated by the T-cellactivation signals, as shown by both phospho-specific immunoblot (IB) and in vitro kinase assays (Fig. 1a,b). Activation of the typical IKK complex by T-cell-activation signals requires a scaffold protein, CARMA1 (refs 16,17). Interestingly, CARMA1 was also required for the activation of TBK1 and IKKe (Fig. 1b). Furthermore, activation of IKKe was completely dependent on IKK, since it was blocked in T cells lacking the IKK regulatory subunit NEMO or the IKK catalytic subunit IKK $\beta$ (Fig. 1b). On the other hand, the activation of TBK1 was only partially inhibited in the NEMO- and IKK $\beta$-deficient $\mathrm{T}$ cells (Fig. 1b). Similar results were obtained using Jurkat $\mathrm{T}$ cells lacking CARMA1 (JPM50.6) (ref. 17) or NEMO (JM4.5.2; ref. 18; Fig. 1c). Thus, both TBK1 and IKKe are activated by T-cell-activation signals, although the underlying mechanism appeared to be different for these kinases.

TBK1 regulates $\mathrm{T}$-cell activation. To study the role of TBK1 in regulating the T-cell function, we generated Tbk1-TKO mice by crossing the Tbk1-floxed mice with CD4-Cre mice. As expected, TBK1 was ablated in T cells but not in B cells (Supplementary Fig. 1a). The T-cell-specific TBK1 ablation did not appreciably alter the pattern of thymocyte development (Supplementary Fig. 1b,c). The young (6 weeks old) Tbk1-TKO and wild-type (WT) control mice also had comparable numbers of $\mathrm{CD}^{+}$and $\mathrm{CD}^{+} \mathrm{T}$ cells in different lymphoid organs (Supplementary a

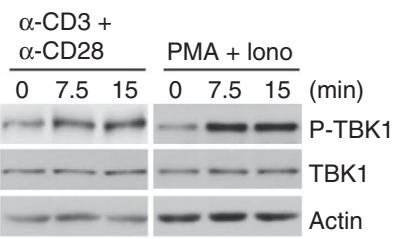

c

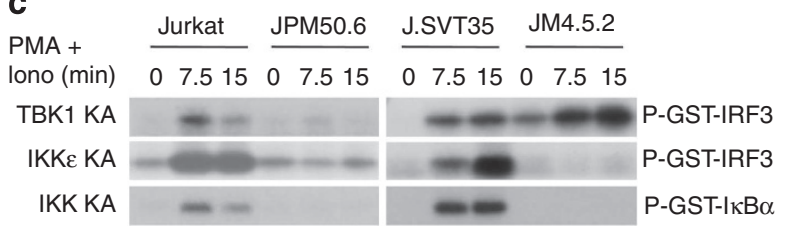

b
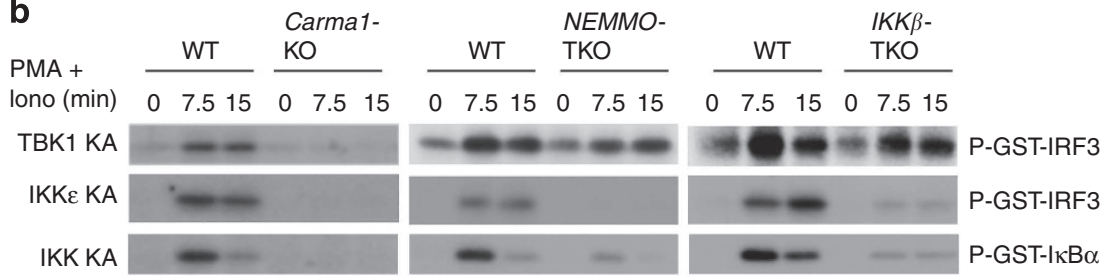

Figure 1 | Activation of TBK1 and IKKe by T-cell activation signals. (a) IB analysis of phosphorylated (P-) TBK1 (Ser172) and total TBK1 in WT $\mathrm{CD}^{+}{ }^{+} \mathrm{T}$ cells (6-week-old mice), stimulated with anti-CD3 plus anti-CD28 using a crosslinking method or with the mitogens PMA plus ionomycin. (b) $\mathrm{CD}^{+}{ }^{+} \mathrm{T}$ cells from the indicated $\mathrm{KO}$ or T-cell-conditional $\mathrm{KO}$ (TKO) and internal WT control mice (6 weeks) were treated with PMA plus ionomycin. TBK1, IKKE and the canonical IKK complex were isolated by IP from the cell lysates and subjected to in vitro kinase assays (KA) using GST-IRF3 (for TBK1 and IKKE) or GST-IKB $\alpha$ (for IKK) recombinant proteins as substrates. (c) TBK1, IKKE and IKK kinase assays were performed using Jurkat and derivative cells as described in $\mathbf{b}$. Data are representative of three or more independent experiments. 
Fig. 1d), suggesting normal survival and homing of naïve $\mathrm{T}$ cells in Tbk1-TKO mice at young ages. Interestingly, however, at an older age (4 months old), the Tbk1-TKO mice had splenomegaly and higher splenocyte numbers (Fig. 2a) as well as increased numbers of $\mathrm{CD}^{+}$and $\mathrm{CD}^{+}{ }^{+} \mathrm{T}$ cells in different lymphoid organs and the peripheral blood (Supplementary Fig. 1e).

To examine the effect of TBK1 deficiency on T-cell activation, we used naïve $\mathrm{CD} 4^{+} \mathrm{T}$ cells derived from the 6-week-old mice. When stimulated in vitro, the WT and TBK1-deficient naïve $\mathrm{CD}^{+} \mathrm{T}$ cells displayed similar level of apoptosis (Supplementary Fig. 1f). Interestingly, however, the TBK1-deficient naive $\mathrm{CD} 4^{+} \mathrm{T}$ cells displayed a higher ability to proliferate in response to lower doses of anti-CD3 (Fig. 2b). Moreover, the stimulated TBK1-deficient $\mathrm{T}$ cells produced remarkably higher levels of IFN- $\gamma$, as detected by both enzyme-linked immunosorbent assay (ELISA) and flow cytometry assays (Fig. 2b,c). On the other hand, the TBK1 deficiency did not substantially promote the expression of two other cytokines, interleukin (IL-2) and granulocytemacrophage colony-stimulating factor (GM-CSF; Fig. 2b,c). Flow cytometry analyses revealed that the Tbk1-TKO mice had a higher frequency of activated or memory-like $\mathrm{T}$ cells, characterized by the CD44hiCD62Llo surface markers, and concomitant reduction in the percentage of naïve
(CD44loCD62Lhi) T cells (Fig. 2d). This phenotype was not detected in mice lacking the TBK1 homologue, IKKE (Fig. 2e), suggesting a unique function of TBK1. Using a Cre-ER-inducible KO approach, we found that TBK1 ablation in adult mice (Supplementary Fig. 2a) also caused an increase in the frequency of activated T cells in the spleen (Supplementary Fig. 2b) without affecting the thymocyte development (Supplementary Fig. 2c,d). Thus, TBK1 appeared to have a non-redundant role in regulating the steady-state activation and homeostasis of $\mathrm{T}$ cells.

Perturbed T-cell homeostasis can be due to a cell-intrinsic mechanism or impaired Treg function. We addressed the mechanism of TBK1 function by mixed bone marrow adoptive transfer. Under such conditions, the WT and Tbk1-TKO T cells were developed and maintained in the same environment and in the presence of WT Treg cells, thus allowing the assessment of cell-intrinsic functions of TBK1. We transferred WT bone marrow from WT mice expressing CD45.1 congenital marker (B6.SJL) along with either WT or Tbk1-TKO bone marrows $\left(\mathrm{CD} 45.2^{+}\right)$into lethally irradiated Rag1-KO mice. As expected, the CD45.1 ${ }^{+}$and $\mathrm{CD} 45.2^{+}$WT T cells in the mixed bone marrow chimeric mice had similar frequency of memory and naïve $\mathrm{CD} 4^{+} \mathrm{T}$ cells (Fig. 2f, left panels). In contrast, the Tbk1TKO $\left(\mathrm{CD} 45.2^{+}\right) \mathrm{CD}^{+} \mathrm{T}$ cells had a profoundly higher a

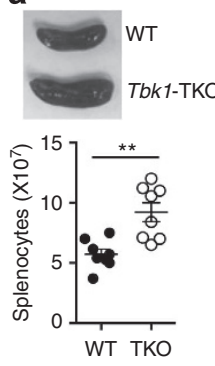

b

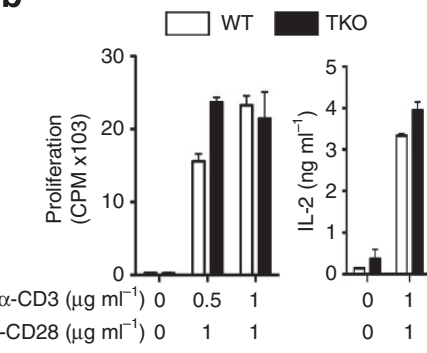

d
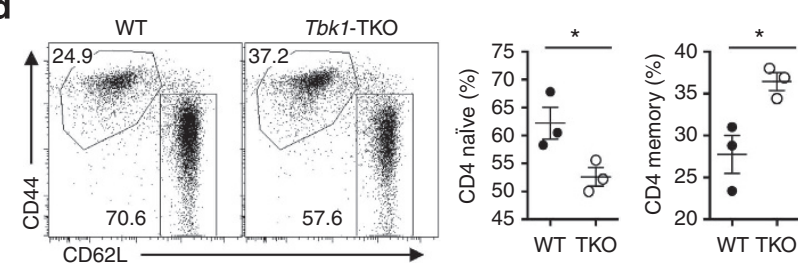

e

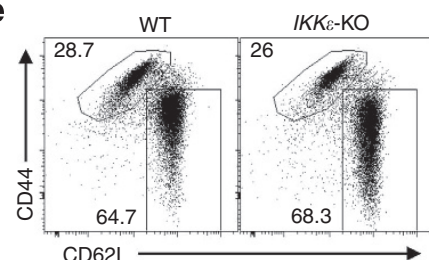

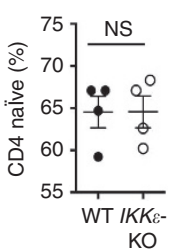

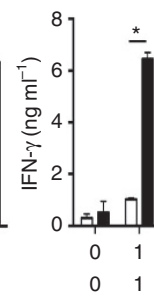

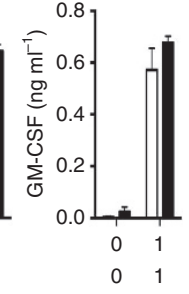
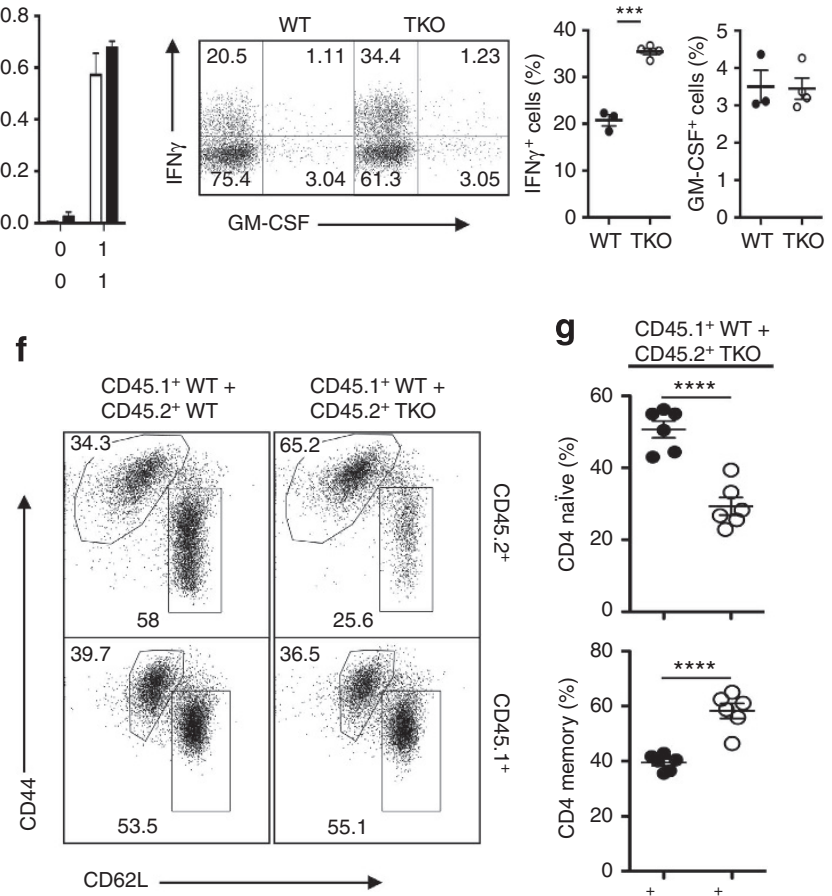
CD45.2+ TKO

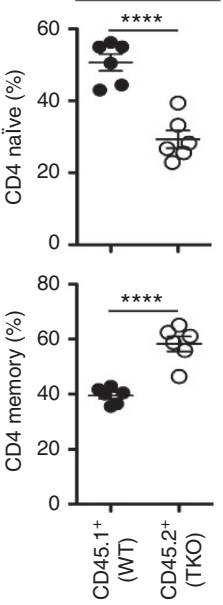

g $\quad \mathrm{CD} 45.1^{+} \mathrm{WT}+$

Figure 2 | TBK1 deficiency promotes T-cell activation and perturbs T-cell homeostasis. (a) A representative spleen picture and mean $\pm \mathrm{s}$.d. values of spleen cell numbers of 4-month-old WT and Tbk1-TKO mice (each circle represents a mouse, $n=8$ ). (b) Proliferation and ELISA (IL-2, IFN- $\gamma$ and GM-CSF) analyses of CD4 ${ }^{+}$T cells purified from the spleen of young ( 6 weeks, $\left.n=4\right)$ WT and TKO mice and stimulated for $48 \mathrm{~h}$ with the indicated concentrations of plate-bound anti-CD3 and anti-CD28. (c) Flow cytometric analyses of splenic CD4 ${ }^{+}$T cells stimulated for $4 \mathrm{~h}$ with PMA and ionomycin in the presence of monensin. Frequency of IFN- $\gamma$-producing and GM-CSF-producing T cells are presented as a representative fluorescence-activated cell sorting plot (left) and mean \pm s.d. values (right) of multiple mice ( 6 weeks, WT $=3$, TKO $=4)$. (d,e) Flow cytometric analyses of the frequency of naïve $\left(\mathrm{CD} 44^{\mathrm{lo}} \mathrm{CD} 62 \mathrm{~L}^{\mathrm{hi}}\right.$ ) and memory (CD44 $\left.{ }^{\mathrm{hi}} \mathrm{CD} 62 \mathrm{~L}^{\mathrm{lo}}\right) \mathrm{CD} 4{ }^{+}$T cells in WT and Tbk7-TKO $(\mathbf{d}, n=3)$ or WT and IKK $\varepsilon$-KO $(\mathbf{e}, n=4)$ mice (6 weeks old). Numbers in quadrant indicate the percentage of cells among total $\mathrm{CD} 4{ }^{+} \mathrm{T}$ cells. Data are representative plot (left) and mean $\pm \mathrm{s}$.d. values (right). (f,g) Flow cytometric analysis of naïve (CD44 $\left.{ }^{\mathrm{lo}} \mathrm{CD} 62 \mathrm{~L}^{\mathrm{hi}}\right)$ and memory $\left(\mathrm{CD} 44^{\mathrm{hi}} \mathrm{CD} 62 \mathrm{~L}^{\mathrm{l}}\right) \mathrm{CD} 4{ }^{+} \mathrm{T}$ cells of lethally irradiated Rag1-KO mice reconstituted with a mixture (1:1 ratio) of bone marrow cells derived from WT B6.SJL $\left(C D 45.1^{+}\right)$and WT or TKO $\left(C D 45.2^{+}\right)$mice $(n=6)$. Data are representative plot $(\mathbf{f})$ and mean \pm s.d. values $(\mathbf{g})$. Data represent mean \pm s.d. from three or more independent experiments. ${ }^{\star} P<0.05 ;{ }^{\star \star} P<0.01 ;{ }^{\star \star \star} P<0.001 ;{ }^{\star \star \star \star} P<0.0001$; NS, non-significant as determined by two-tailed unpaired Student's $t$-test, comparing the indicated groups. 
frequency of memory population compared with the WT $\left(\mathrm{CD} 45.1^{+}\right) \mathrm{CD}^{+} \mathrm{T}$ cells within the same chimeric mice (Fig. 2f, right panels, and Fig. 2g). These data suggest that TBK1 has a cell-intrinsic role in the regulation of steady-state activation and homeostasis of $\mathrm{T}$ cells.

TBK1 is essential for EAE induction. Since the reduced threshold of T-cell activation often promotes autoimmunity, we examined the role of TBK1 in regulating a T-cell-dependent autoimmunity, EAE. The pathogenesis of EAE involves the inflammatory action of CD4 ${ }^{+} \mathrm{T}$ helper 17 (Th17) and Th1 cells, which are characterized by the production of the signature cytokines IL-17 and IFN- $\gamma$, respectively ${ }^{3}$. Immunization of WT mice with a myelin-specific autoantigen, myelin oligodendrocyte glycoprotein (MOG) peptide $\mathrm{MOG}_{35-55}$, led to the induction of overt EAE clinical scores (Fig. 3a). Surprisingly, the Tbk1-TKO mice were not hypersensitive, but rather remarkably resistant, to EAE induction (Fig. 3a). Histological analyses revealed drastic reduction in CNS-infiltrating immune cells and $\mathrm{CD} 4{ }^{+} \mathrm{T}$ cells in the Tbk1-TKO mice (Fig. 3b, left and middle). In addition, consistent with the clinical scores, the spinal cord of the WT, but not Tbk1-TKO, mice had severe demyelination (Fig. 3b, right). Parallel flow cytometry analyses confirmed the drastic reduction in the number of $\mathrm{CD}^{+}{ }^{+}$and $\mathrm{CD} 8{ }^{+} \mathrm{T}$ cells in the CNS of EAEinduced Tbk1-TKO mice (Fig. 3c). The EAE-refractory phenotype of the Tbk1-TKO mice was unlikely due to a defect in inflammatory $\mathrm{T}$-cell production, since the TBK1-deficient $\mathrm{CD} 4{ }^{+} \mathrm{T}$ cells generated higher levels of Th1 and Th17 cells than WT $\mathrm{CD}^{+}{ }^{+} \mathrm{T}$ cells in an in vitro differentiation assay (Supplementary Fig. 3). Consistently, despite the drastically reduced T-cell numbers in the CNS of Tbk1-TKO mice, the percentage of the effector T cells expressing IFN- $\gamma$ (Th1), IL-17A (Th17) or GM-CSF within the CD4 ${ }^{+} \mathrm{T}$-cell population in the CNS, spleen or draining lymph node of Tbk1-TKO mice was either higher than or similar to that of the WT control mice (Fig. 3d-f). Notably, the absolute number of the inflammatory effector $\mathrm{CD} 4^{+} \mathrm{T}$ cells was drastically increased in the draining lymph nodes of the Tbk1-TKO mice due to the accumulation of total $\mathrm{CD}^{+}{ }^{+} \mathrm{T}$ cells (Fig. 3g). These results suggest that although TBK1 deficiency promotes T-cell activation and differentiation, it attenuates EAE induction probably by blocking the migration of the effector T cells from the draining lymph nodes to the CNS.

To further investigate the EAE-regulating function of TBK1, we examined the role of TBK1 in regulating migration of autoimmune T cells to the CNS. Since TBK1 deficiency did not decrease the cellularity or subset composition of $\mathrm{T}$ cells in the lymph nodes (Supplementary Fig. 1d,e), it is likely that TBK1 is a

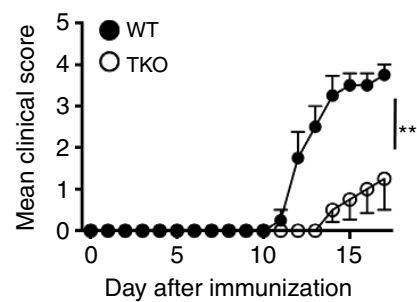

b

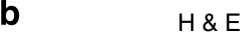

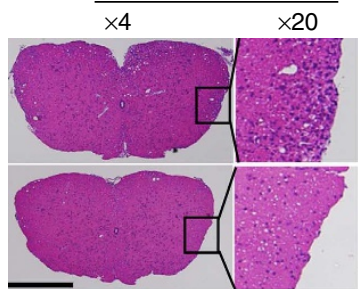

$\alpha-\mathrm{CD} 4 \mathrm{IHC}$
$\times 4$
$\times 20$

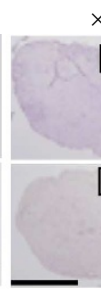

$\times 20$

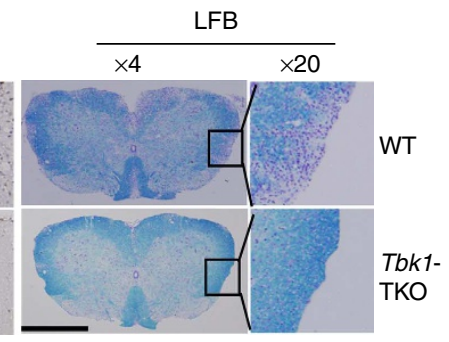

c

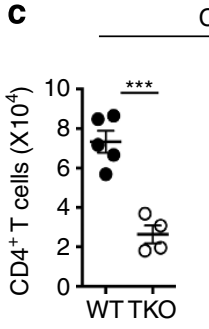

CNS

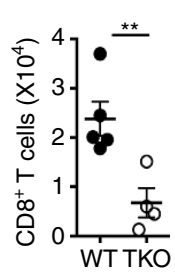

d

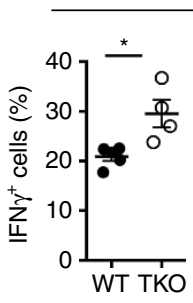

CNS

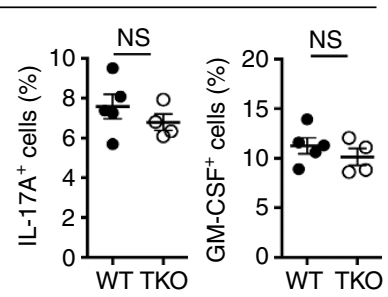

e

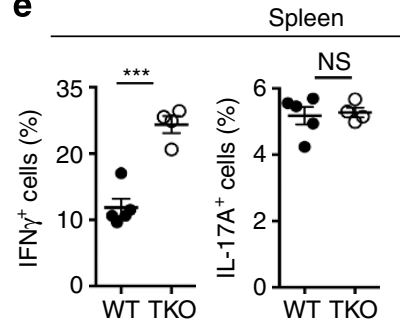

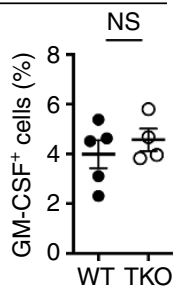

f

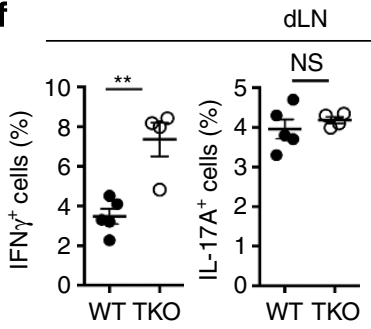

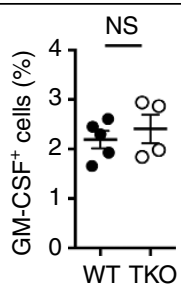

g

dLN

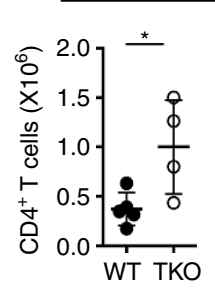

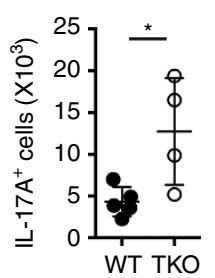

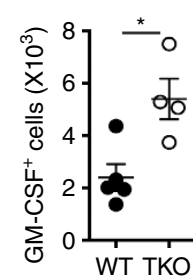

Figure 3 | TBK1 is crucial for EAE induction. (a) EAE disease score of WT $(n=5)$ and Tbk1-TKO $(n=4)$ mice. (b) Haematoxylin and eosin (H\&E), CD4 $\mathrm{IHC}$, and Luxol Fast Blue (LFB) staining of spinal cord sections from $\mathrm{MOG}_{35-55}$-immunized WT and TKO EAE mice (28 day after immunization). Scale bars, $500 \mu \mathrm{m}$. Data are representative of three independent experiments. (c) Flow cytometry determining the mean \pm s.d. values of CD4 ${ }^{+} \mathrm{T}$-cell number and $\mathrm{CD} 8{ }^{+}$T-cell number recovered from the CNS of WT and Tbk7-TKO mice 18 days after EAE induction. (d-f) Flow cytometric analyses of CD4 ${ }^{+}$ T cells from CNS, spleen and lymph nodes of the EAE-induced mice described in a (18 days after EAE induction). The T cells were stimulated for $4 \mathrm{~h}$ with PMA and ionomycin in the presence of monensin, followed by flow cytometry analysis of the IFN- $\gamma$, IL-17A and GM-CSF-producing cells. (g) Mean \pm s.d. values of absolute numbers of IFN- $\gamma$, IL-17A- and GM-CSF-producing CD4 ${ }^{+}$T cells recovered from the draining lymph node (dLN) of WT or Tbk1-TKO mice described in a. Data represent mean \pm s.d. from three or more independent experiments. ${ }^{\star} P<0.05 ;{ }^{\star \star} P<0.01$; ${ }^{\star \star \star} P<0.001$; NS, non-significant as determined by two-way analysis of variance with Bonferroni's post test for EAE clinical scores analysis and two-tailed unpaired Student's $t$-test for other analysis, comparing the indicated groups. 
dispensable for T-cell homing to the lymph nodes. Indeed, adoptively transferred Tbk1-TKO CD4 ${ }^{+} \mathrm{T}$ cells efficiently homed to the spleen and lymph nodes in the T-cell-deficient Tcrb/d-KO mice (Fig. 4a). In fact, T-cell cellularity in Tbk1-TKO mice was moderately increased in the lymphoid organs and decreased in the peripheral blood, compared with WT mice (Fig. 4a). Thus, although the Tbk1-TKO mice displayed reduced frequency of CD62L ${ }^{\text {hi }} \mathrm{T}$ cells (Fig. 2d), they did not have a defect in T-cell homing to the lymph nodes as shown for the CD62Ldeficient mice ${ }^{19,20}$.

T-cell migration into the CNS is promoted by the inflammatory CNS microenvironment. To test whether the defect of the Tbk1-TKO T cells in CNS infiltration was due to the cell-intrinsic mechanisms, we employed a mixed bone marrow adoptive transfer approach. We adoptively transferred a mixture of green fluorescent protein $\left(\mathrm{GFP}^{+}\right)$WT bone marrows and $\mathrm{GFP}^{-} \mathrm{Tbk1-}$ TKO bone marrows into lethally irradiated Rag1-KO mice and induced EAE in the recipient mice. On the basis of GFP expression, we analysed the frequency of WT and Tbk1-TKO
$\mathrm{T}$ cells in the CNS and draining lymph nodes of the recipient mice during the early effector phase (day 14) of EAE induction. Within the same recipient mice, the WT $\mathrm{T}$ cells $\left(\mathrm{GFP}^{+}\right)$ efficiently migrated to the CNS, whereas the majority of the Tbk1-TKO T cells $\left(\mathrm{GFP}^{-}\right)$were retained in the draining lymph nodes (Fig. $4 \mathrm{~b}$ ). Since the WT and TBK1-deficient $\mathrm{T}$ cells were activated under the same conditions, these results suggest that the defect of the TBK1-deficient T cells in infiltrating into CNS is not due to attenuated CNS inflammation but rather due to a cellintrinsic deficiency.

TBK1 deficiency promotes T-cell retention in lymph nodes. Antigen-activated $\mathrm{T}$ cells are initially retained in draining lymph nodes to undergo differentiation and subsequently exit the lymph nodes and migrate to the peripheral site of infection or inflammation ${ }^{21}$. Since the TBK1-deficient $\mathrm{T}$ cells were accumulated in the draining lymph nodes during EAE induction (Fig. 4b), we examined whether the loss of TBK1 affected T-cell egress from
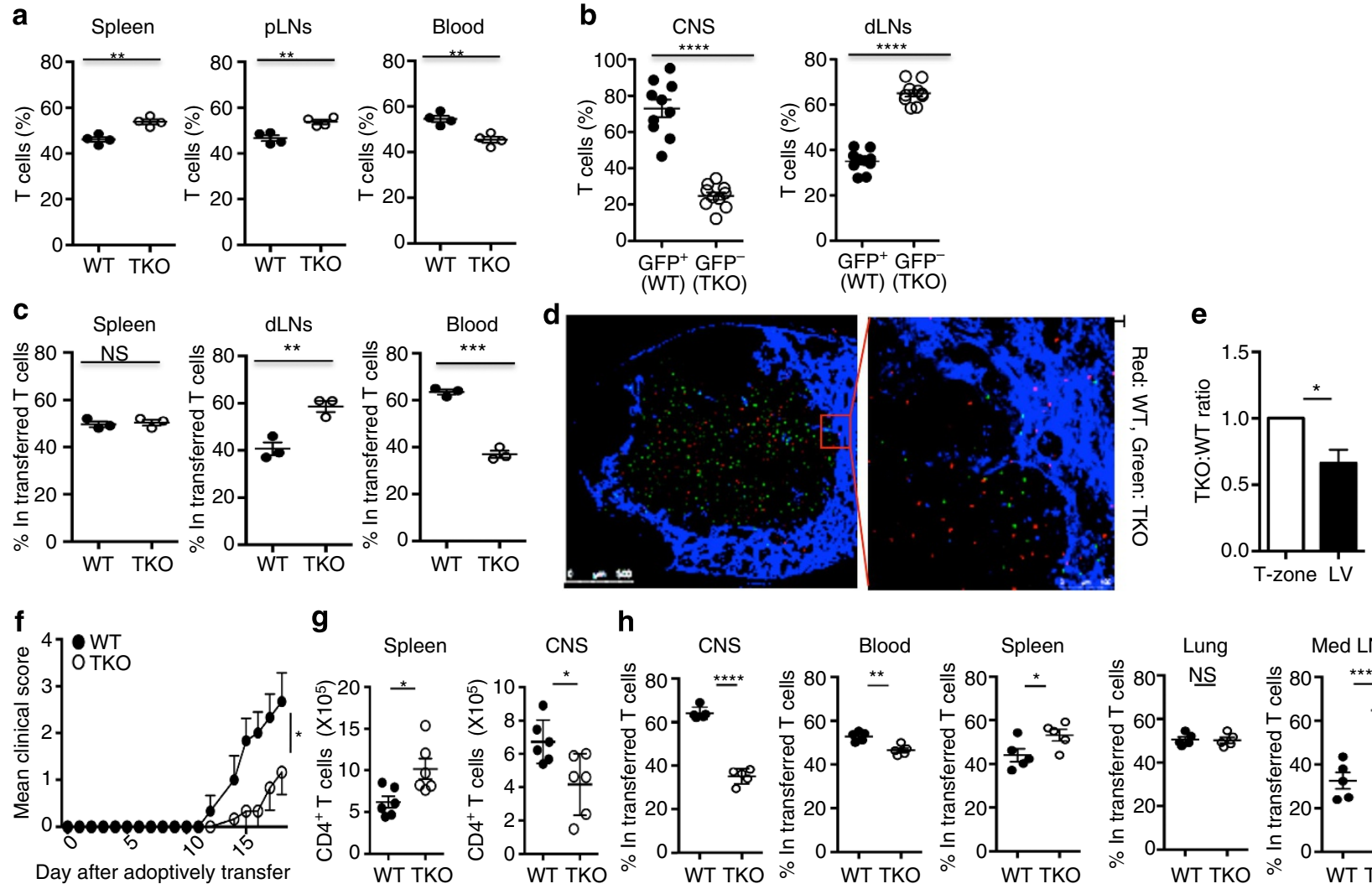

$\mathbf{h}$
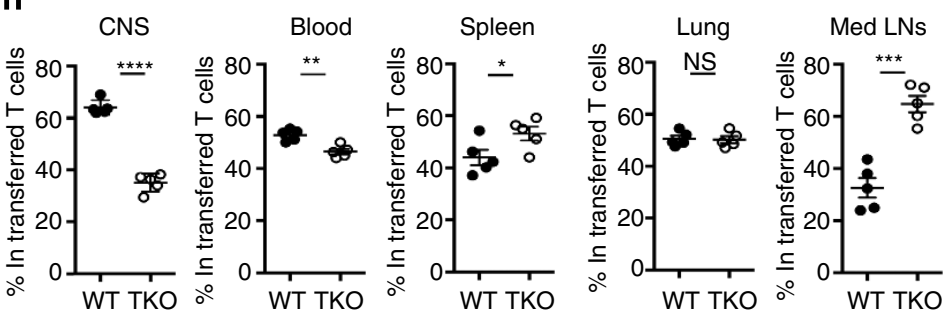

Figure 4 | TBK1 deficiency promotes T-cell retention in draining lymph nodes. (a) WT and Tbk1-TKO naïve CD4 ${ }^{+}$cells were labelled with CMRA and CFSE, respectively, and adoptively transferred into $\mathrm{Tcrb} / \mathrm{d}-\mathrm{KO}$ mice in a mixture (at 1:1 ratio). Cell distribution in different organs was analysed $16 \mathrm{~h}$ later by flow cytometry $(n=4)$. (b) Lethally irradiated Rag1-KO mice were reconstituted with a mixture (1:1 ratio) of bone marrow cells isolated from Tbk1-TKO and GFP-expressing WT mice $(n=10)$. The chimeric mice were immunized with $\mathrm{MOG}_{35-55} 6$ weeks after reconstitution. CNS and draining LN (dLN) CD4 ${ }^{+}$ T cells were analysed based on GFP expression gated on CD4 ${ }^{+}$cell on day 14 after EAE induction. (c-e) WT and Tbk1-TKO naïve CD4 ${ }^{+}$cell were labelled with CMRA or CFSE respectively and adoptively transferred into WT mice at 1:1 ratio, followed by MOG $_{35-55}$ immunization. Five days later, recipient mice were subjected to flow cytometry analysis of cell distribution in different organs (c) or confocal imaging of the differentially labelled T cells in a draining LN, showing the entire T-cell zone (left) and the peripheral region (right) (d, red, CMRA-labelled WT T cells; green, CFSE-labelled Tbk1-TKO T cells; blue, LYVE-1+ lymphatic vessels). TKO:WT T-cell ratio in the T-cell zone (set at an index of 1:1) and lymphatic vessel are presented as mean \pm s.d. values of based on three experiments with a total of $>500$ T cells analysed $(\mathbf{e})$. (f,g) Clinical scores (f) and flow cytometry analysis of CD4 ${ }^{+} \mathrm{T}$ cells $(\mathbf{g}$, day 18$)$ of Tcrb/d-KO mice undergoing passive EAE induction after being adoptively transferred with WT or Tbk1-TKO MOG-primed CD4 ${ }^{+}$T cells. (h) Flow cytometry analysis of $\mathrm{CD}^{+}{ }^{+}$T cells in the indicated organs and tissues of WT mice adoptively transferred (for 4 days) with a mixture (1:1 ratio) of MOG-primed WT (CMRA labelled) and Tbk1-TKO (CFSE-labelled) CD4 ${ }^{+}$T cells (1:1 ratio). Data represent mean $\pm \mathrm{s}$.d. from two or more independent experiments. ${ }^{\star} P<0.05 ;{ }^{\star \star} P<0.01$; ${ }^{\star \star \star} P<0.001 ;{ }^{\star \star \star \star} P<0.001$; NS, non-significant as determined by two-way analysis of variance with Bonferroni's post test for EAE clinical scores analysis and two-tailed unpaired Student's $t$-test for other analysis, comparing the indicated groups. 
the lymph nodes, by employing a mixed T-cell transfer approach. We labelled the WT and Tbk1-TKO naïve $\mathrm{CD} 4{ }^{+} \mathrm{T}$ cells with CellTracker Orange CMRA dye and carboxyfluorescein succinimidyl ester (CFSE) fluorescence dyes, respectively, and transferred a mixture of the labelled cells (in 1:1 ratio) into WT mice. We then analysed the distribution of the WT and Tbk1TKO T cells during an early phase of MOG immunization (day 5). We detected the accumulation of Tbk1-TKO T cells in the draining lymph nodes with concomitantly reduced frequency in the peripheral blood (Fig. 4c). This result indicated enhanced lymph node retention of the TBK1-deficient $\mathrm{T}$ cells following antigen stimulation. In parallel, we also performed confocal imaging to analyse the $\mathrm{T}$ cells in both the T-cell zone and the lymphatic vessel region. Consistent with the flow cytometry analysis result (Fig. 4c), the CFSE-labelled Tbk1-TKO T cells were clearly more abundant than the CMRA-labelled WT T cells in the T-cell zone of the draining lymph node (Fig. 4d, left). Interestingly, however, a significantly lower TKO:WT T-cell ratio was found in the lymphatic vessel area compared with that in T-cell zones (Fig. 4d, right; Fig. 4e), consistent with a reduced egress of TKO T cells through the cortical sinuses (Fig. 4d,e). These results indicate that TBK1 may regulate the exit of effector $\mathrm{T}$ cells from the draining lymph nodes.

Since the TBK1 deficiency only partially affected the lymph node exit of T cells, we wondered whether the Tbk1-TKO mice were competent in mounting immune responses against infections. In an influenza viral infection model, we found that the lung of the virus-infected Tbk1-TKO mice only had moderately reduced frequency of $\mathrm{CD}^{+}$and $\mathrm{CD} 8{ }^{+} \mathrm{T}$ cells and increased frequency of $\mathrm{T}$ cells in the draining mediastinal lymph nodes (Supplementary Fig. 4a). Both the body weight loss (Supplementary Fig. $4 \mathrm{~b}$ ) and the cytokine concentration in bronchoalveolar lavage were comparable between the WT and Tbk1-TKO mice (Supplementary Fig. 4c). These results suggest competent immune responses to influenza viral infection in the Tbk1-TKO mice.

To understand why the TBK1 deficiency particularly influenced the pathogenesis of EAE, we performed passive EAE studies. We isolated T cells from MOG-immunized WT or Tbk1TKO mice and restimulated them in vitro using the MOG peptide. After expansion, we adoptively transferred the autoimmune $\mathrm{WT}$ and Tbk1-TKO effector T cells intravenously (i.v.) into Tcrb/d-KO mice. Interestingly, under these conditions that would bypass the step of T-cell exit from the draining lymph node, the TBK1-deficient $\mathrm{T}$ cells were still significantly defective in inducing EAE (Fig. 4f), coupled with reduced CD4 T-cell number in the CNS and increased CD4 T-cell number in the spleen on day 18 following the T-cell transfer (Fig. 4g). Gene expression analysis revealed that the Tbk1-TKO T cells had a moderate reduction in the expression of Ninj1 gene and competent expression of several other cell adhesion molecules and chemokine receptors implicated in T-cell migration into the CNS (Supplementary Fig. 5) 22-24. To further assess the mechanism by which TBK1 mediates CNS infiltration of $\mathrm{T}$ cells, we compared the migration of the WT and TBK1deficient $\mathrm{T}$ cells by labelling the in vitro-expanded MOG-primed WT and Tbk1-TKO T cells with different fluorescence dyes (CMRA and CFSE, respectively) and transferring them in a mixture (1:1 ratio) into WT mice. We then analysed the frequency of the WT and Tbk1-TKO T cells (in the same recipients) in the blood, lung and lymphoid organs during the early phase (day 4) of T-cell transfer. This approach further confirmed the defect of the Tbk1-TKO effector T cells in migrating to the CNS (Fig. 4h). Interestingly, the Tbkl-TKO effector T cells were accumulated in the mediastinal lymph nodes (Fig. 4h). Of note, autoimmune $\mathrm{T}$ cells are known to migrate to the CNS via the lung and the lung-draining mediastinal lymph nodes ${ }^{24}$. These results suggest that the impaired CNS infiltration by TBK1-deficient autoimmune $\mathrm{T}$ cells may involve increased intranodal retention in different steps, although the involvement of TBK1 in regulating some other aspects of T-cell migration is also possible.

TBK1 controls activation of AKT and mTORC1. To understand the signalling mechanism by which TBK1 regulates T-cell homeostasis and EAE pathogenesis, we examined the effect of the T-cell-specific TBK1 ablation on the activation of signalling molecules. A striking phenotype of the Tbk1-TKO T cells was elevated homeostatic activation of AKT (Fig. 5a). The phosphorylation of Foxo1, a known target of $\mathrm{AKT}^{25}$, was also higher in the TBK1-deficient $\mathrm{T}$ cells (Fig. 5a). Another major downstream target of AKT is the metabolic kinase mTORC1 (ref. 26). Consistently, the TBK1-deficient T cells had elevated phosphorylation of the mTORC1 substrate protein S6 kinase 1 (S6K1) as well as the S6K1 substrate S6 (Fig. 5a). In contrast, the loss of TBK1 did not alter the phosphorylation of the MAP kinases p38 and ERK (Supplementary Fig. 6a). The crucial role of TBK1 in controlling the homeostatic activation of AKT and mTORC1 was unique, since it was not seen with the IKKe-KO T cells (Supplementary Fig. 6b). Furthermore, the deregulated signalling events in the Tbk1-TKO T cells were not due to their activation state, since similar results were obtained with both naïve and memory $\mathrm{CD} 4^{+} \mathrm{T}$ cells (Supplementary Fig. 6c). The loss of TBK1 also promoted the phosphorylation of AKT and S6 stimulated by anti-CD3 plus anti-CD28 (Fig. 5b). Moreover, we detected hyperphosphorylation of AKT as well as S6 and S6K1 in Tbk1-TKO T cells isolated during the effector phase of EAE induction (Fig. 5c). Using the Cre-ER-inducible KO system, we found that TBK1 ablation in adult mice also caused aberrant phosphorylation of AKT and S6 in T cells (Supplementary Fig. 6d), further emphasizing a pivotal role for TBK1 in controlling the activation of AKT and its downstream targets, Foxo1 and mTORC1.

TBK1 induces phosphorylation-dependent AKT degradation. Although AKT activation has been extensively studied, how this master kinase is negatively controlled is unclear. We found that the hyperactivation of AKT in TBK1-deficient T cells was associated with a profound increase in the level of total AKT protein (Fig. 5a and Supplementary Fig. 6b-d). This phenotype was not due to enhanced AKT mRNA expression (Supplementary Fig. 6e), indicating a post-translational mechanism of AKT regulation. We thus examined the possible involvement of the ubiquitin-proteasome pathway. Because of the low number of naive $\mathrm{T}$ cells in the Tbk1-TKO mice, we used thymocytes for AKT ubiquitination assays. As seen in peripheral T cells, the TBK1deficient thymocytes also had elevated level of AKT (Fig. 5d). Interestingly, AKT was undergoing lysine 48-linked polyubiquitination in the WT thymocytes, which was drastically reduced in the TBK1-deficient thymocytes (Fig. 5d). This finding suggests that TBK1 controls the homeostatic level of AKT by promoting ubiquitin-dependent AKT degradation, thus explaining the accumulation of AKT in the TBK1-deficient T cells.

Since the homeostasis of $\mathrm{T}$ cells involves constant stimulation of the TCR by self-ligands ${ }^{27}$, we asked whether the TCR signal could stimulate AKT degradation and whether this signalling event was regulated by TBK1. To avoid the homeostatic effect of TBK1 deficiency, we employed WT $\mathrm{CD}^{+}{ }^{+} \mathrm{T}$ cells. Indeed, the stimulation of the WT $\mathrm{CD}^{+}{ }^{+} \mathrm{T}$ cells with anti-CD3 and anti-CD28 led to the gradual loss of AKT (Fig. 5e). Moreover, a small-molecule inhibitor of TBK1, MRT67307 (ref. 28), efficiently inhibited the AKT degradation (Fig. 5e). We next employed a 
a

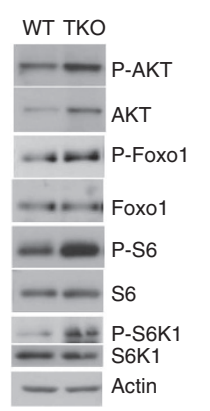

b

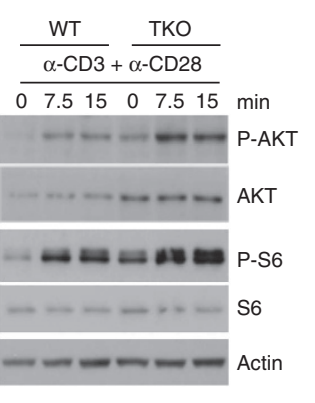

C

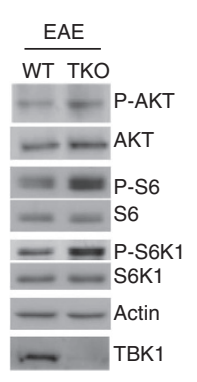

d

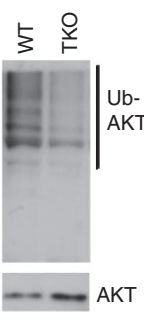

e

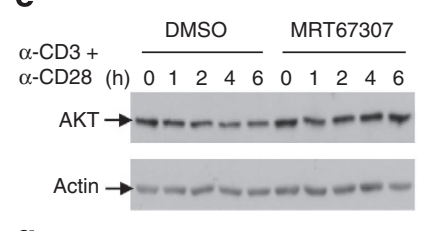

9 HA-AKT WT T195A T308A S378A FLAG-TBK1 $\overline{-+} \overline{-+} \overline{-+}$ HA-AKT $\rightarrow-\cdots-\cdots-$

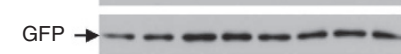

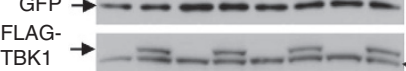

\section{f}

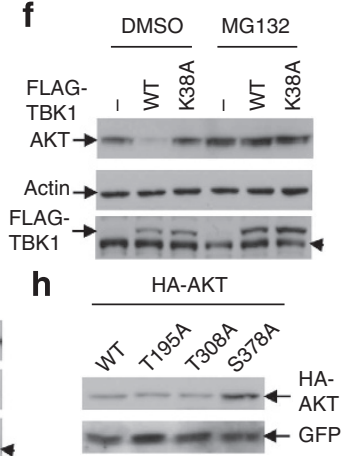

i

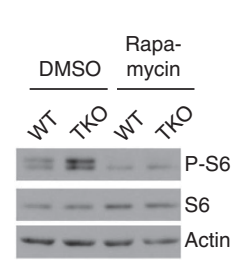

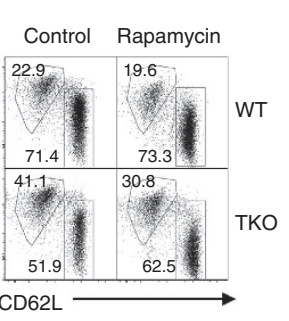

j

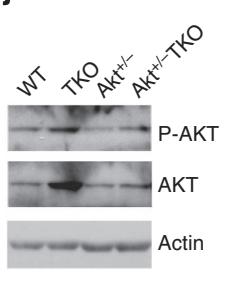

k

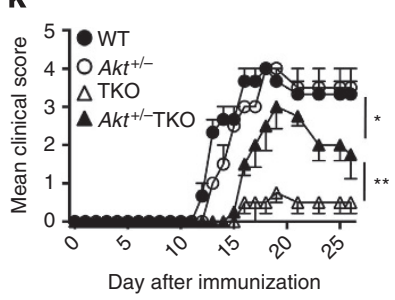

I

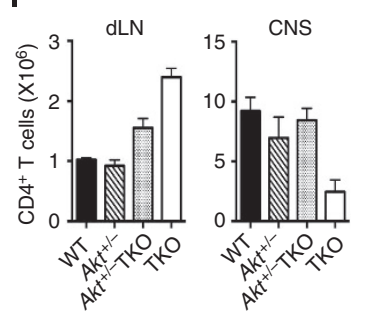

m

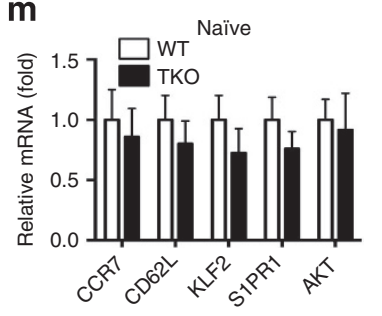

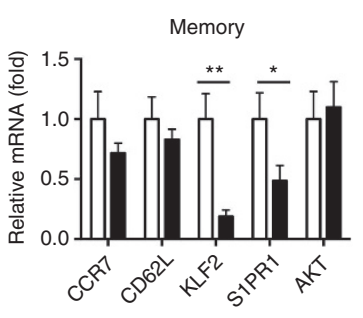

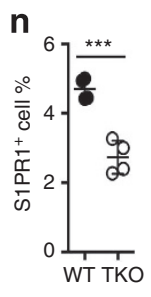

o

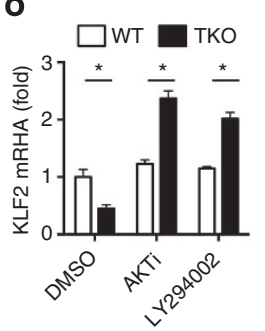

Figure 5 | TBK1 stimulates AKT degradation and negatively regulates the activation of AKT and mTORC1 pathways. (a) IB analysis of phosphorylated (P-) AKT (Ser473), Foxo1 (Thr24), S6 (Ser235/236), S6K1 (Thr389), as well as their total protein controls in CD4 ${ }^{+}$T cells from WT and Tbk1-TKO mice (6 weeks old). (b) IB analysis of the indicated phosphorylated (P-) and total proteins in CD4 ${ }^{+} \mathrm{T}$ cells activated by anti-CD3 plus anti-CD28 using a crosslinking method. (c) IB analysis of the indicated phosphorylated (P-) and total proteins in CD4 ${ }^{+}$T cells isolated from day 18 EAE-induced WT or Tbk1TKO mice. (d) AKT was isolated by IP from the lysates of thymocytes derived from WT or Tbk1-TKO mice (6 weeks old), and the ubiquitinated AKT (Ub-AKT) was detected by IB using an anti-ubiquitin antibody detecting K48-linked polyubiquitin chains. (e) IB analysis using WT CD4 ${ }^{+}$T cells, pretreated for $1 \mathrm{~h}$ with a TBK1 inhibitor, MRT67307, of solvent control dimethylsulphoxide (DMSO) and subsequently stimulated for the indicated times with anti-CD3 plus anti-CD28 in the presence of a protein synthesis inhibitor, cycloheximide. (f) IB analysis of endogenous AKT and Actin and exogenous FLAG-TBK1 in whole-cell lysates of HEK293T cells, transfected as indicated and subsequently incubated for $2 \mathrm{~h}$ with DMSO or MG132 (25 $\mu$ M). An arrowhead indicates the non-specific band. (g) IB analysis of the indicated exogenous proteins in the whole-cell lysates of HEK293 cells transfected with HA-AKT WT or the indicated point mutants either in the presence $(+)$ or absence $(-)$ of FLAG-tagged TBK1. An arrowhead indicates the non-specific band. (h) IB analysis of whole-cell lysates of WT CD4 ${ }^{+}$T cells transduced with pMIGR1 retroviruses encoding WT or mutant forms of AKT as well as the GFP marker. (i) IB (left) and flow cytometry (right) analyses of splenic CD4 ${ }^{+}$T cells from WT or Tbk1-TKO mice treated injected (i.p) with rapamycin for 5 days $(n=5)$. (j) IB analysis of $C D 4^{+}$cells from spleen of the indicated mouse strains. (k,I) EAE disease score $(\mathbf{k})$ and CD4 ${ }^{+}$T-cell number of draining LN and CNS (I, day27) (I) of the indicated mouse strains, immunized for EAE induction $(n=4)$. ( $\mathbf{m})$ Quantitative PCR (qPCR) analysis of the indicated genes in freshly isolated naïve or memory CD4 ${ }^{+}$T cells from WT and Tbk1-TKO mice $(n=4)$. ( $(\mathbf{n})$ S1PR1 flow cytometry analysis in memory CD4 ${ }^{+}$cell of inguinal LN from WT or Tbk1-TKO mice. (o) qPCR analysis of KLF2 in CD4 ${ }^{+}$T cells treated for $1 \mathrm{~h}$ with either DMSO control or inhibitors for AKT (AKTi) or PI3 kinase (LY294002). Data represent mean \pm s.d. from two or more independent experiments. ${ }^{\star} P<0.05 ;{ }^{\star \star} P<0.01$; NS, non-significant as determined by two-way analysis of variance with Bonferroni's post test for EAE clinical scores analysis and two-tailed unpaired Student's $t$-test for other analysis, comparing the indicated groups.

transient transfection model to see whether overexpressed TBK1 was able to induce AKT degradation. Indeed, the expression of TBK1, but not its catalytically inactive mutant (K38A), caused drastic loss of endogenous AKT, which was blocked by a proteasome inhibitor, MG132 (Fig. 5f). TBK1 also induced the proteolytic degradation of exogenously expressed AKT but did not affect the co-transfected control protein GFP (Supplementary Fig. 6f).

A previous study suggests that the overexpressed TBK1 phosphorylates AKT at threonine-195 and serine-378, but the functional significance has not been determined ${ }^{29}$. Our finding that TBK1 stimulated AKT degradation led us to examine whether these phosphorylation sites regulate TBK1 stability. Mutation of threonine-195, or the activation-loop phosphorylation site threonine-308, of AKT only weakly inhibited TBK1-stimulated degradation (Fig. 5g). Interestingly, the mutation of serine-378 of AKT completely prevented its degradation by TBK1 (Fig. 5g). When stably expressed in T cells, the AKT mutant harbouring the serine-378 mutation also displayed markedly higher level of expression (Fig. 5h). These results indicate that the TBK1-induced AKT phosphorylation at serine-378 plays an important role in regulating the fate of AKT. 
Functional significance of AKT-mTORC1 deregulation. Our finding that TBK1 controls the activation of AKT and mTORC1 provides molecular insight into the impaired T-cell homeostasis in Tbk1-TKO mice, since the AKT-mTORC1 signalling axis has a crucial role in the regulation of $\mathrm{T}$-cell homeostasis ${ }^{26}$. Indeed, we found that treatment of Tbk1-TKO mice with an mTORC1 inhibitor, rapamycin, blocked the homeostatic activation of mTORC1 and corrected the T-cell homeostasis phenotype (Fig. 5i).

It is known that activated $\mathrm{T}$ cells initially downregulate homing receptors, particularly sphingosine-1-phosphate receptor 1 (S1PR1), and then regain the expression of S1PR1 for migrating out of the lymph nodes ${ }^{21}$. This dynamic change is coupled with the initial activation of $\mathrm{AKT}$ and the subsequent decline of the AKT activity. Since Tbk1-TKO T cells had aberrant AKT accumulation and activation, we tested whether the ablation of AKT could correct the EAE phenotype of the Tbk1-TKO mice. We crossed the Tbk1-TKO mice with Akt-KO mice to generate $A k t^{+/-}$Tbk1-TKO mice. Interestingly, ablation of one allele of $A k t$, which reduced the level of AKT and P-AKT in Tbk1-TKO $\mathrm{T}$ cells (Fig. 5j), largely restored the sensitivity of the Tbk1-TKO mice to EAE induction (Fig. 5k) as well as the CNS migration of the Tbk1-TKO T cells (Fig. 5l).

A hallmark of the CNS-migrating autoimmune $\mathrm{T}$ cells is the high level expression of the transcription factor KLF2 and its target gene S1PR1, the latter of which has become an attractive target for MS therapy ${ }^{24,30,31}$. In naïve CD4 ${ }^{+} \mathrm{T}$ cells, the TBK1 deficiency only moderately reduced the expression of KLF2 and S1PR1 as well as two other T-cell homing genes, CCR7 and CD62L (Fig. 5m). Interestingly, however, the TBK1 deficiency greatly inhibited the expression of KLF2 and S1PR1, although only moderately reduced expression of CCR7 and CD62L, in the memory population of $\mathrm{CD}^{+}{ }^{+} \mathrm{T}$ cells (Fig. $5 \mathrm{~m}$ ). Flow cytometry analyses also revealed reduced level of surface expression of S1PR1 in Tbk1-TKO CD $4^{+} \mathrm{T}$ cells (Fig. $5 \mathrm{n}$ ).

The expression of KLF2 is known to be mediated by Foxo1, a transcription factor that is negatively regulated by $\mathrm{AKT}^{32}$. Interestingly, incubation of Tbk1-TKO CD4 ${ }^{+} \mathrm{T}$ cells with small-molecule inhibitors for AKT (AKTi) and its upstream regulator PI3 kinase (LY294002) greatly promoted the expression of KLF2 (Fig. 5o). On the other hand, these inhibitors only slightly increased the expression of KLF2 in the WT T cells (Fig. 5o), consistent with the low basal AKT activity in WT T cells (Fig. 5a). Thus, the TBK1 deficiency attenuated the expression of KLF2 and S1PR1, which in turn appeared to be due to the deregulated AKT activation. Collectively, these data indicate that the aberrant AKT activation in TBK1-deficient $\mathrm{T}$ cells contributes to their attenuated CNS migration and induction of EAE.

To examine the role of TBK1 in regulating AKT-mTORC1 signalling and gene expression in human T cells, we silenced the expression of TBK1 using short hairpin RNAs (shRNAs) expressed in a lentiviral vector. Interestingly, shRNA-mediated TBK1 silencing promoted the phosphorylation of AKT as well as the AKT-mTORC1 downstream proteins Foxol and S6 (Supplementary Fig. 7a). Consistently, the TBK1 knockdown also reduced the expression of S1PR1 and KLF2 as well as CD62L (Supplementary Fig. 7b). Using a well-defined in vitro T-cell migration model ${ }^{33}$, we found that TBK1 knockdown in human $\mathrm{CD}^{+} \mathrm{T}$ cells significantly inhibited their ability to transmigrate through a human brain microvascular endothelial cell (EC) monolayer (Supplementary Fig. 7c). Similar results were obtained when the T cells were treated with a TBK1 inhibitor, amlexanox (Supplementary Fig. 7d).

In parallel with the functional studies, we analysed the potential alterations of TBK1 expression in MS patients. Our data revealed that the expression of TBK1 is significantly increased in the peripheral blood mononuclear cell (PBMC) of MS patients compared with the healthy donors (Supplementary Fig. 7e). Consistently, TBK1 expression was also shown to be elevated (2.41- and 1.79-folds) in two MS PBMC microarray databases ${ }^{34,35}$. These results suggest that TBK1 controls the AKTmTORC1 signalling axis in both murine and human $\mathrm{T}$ cells.

A TBK1 inhibitor ameliorates EAE pathogenesis. The data described above not only revealed a previously unknown signalling mechanism regulating T-cell function and CNS inflammation but also implicated TBK1 as an attractive therapeutic target for the treatment of MS. To further assess the therapeutic value of TBK1, we tested the effect of TBK1 pharmacological inhibition on the induction of EAE. In this regard, a recent study identified the Food and Drug Administration-approved therapeutic compound amlexanox as a selective inhibitor of TBK1 (ref. 36). We asked whether amlexanox could ameliorate EAE and, if so, whether it acted through modulating T-cell migration into the CNS. We treated the mice daily with amlexanox (via intraperitoneal (i.p.) injection) during the induction of EAE. The amlexanox treatment drastically delayed the onset, and reduced the severity, of the EAE disease (Fig. 6a), which was associated with a profound reduction in the number of $\mathrm{CD}^{+}$and $\mathrm{CD}^{+}{ }^{+} \mathrm{T}$ cells in the CNS of the EAE-induced mice (Fig. 6b). As seen with the TBK1 gene ablation, the amlexanox treatment did not inhibit $\mathrm{T}$-cell expansion but rather caused the sequestration of $\mathrm{T}$ cells in the draining lymph nodes and spleen (Fig. 6c). Moreover, the T cells isolated from the amlexanox-treated mice displayed aberrant activation of AKT and its target protein Foxol as well as the mTOCR1 pathway component S6 (Fig. 6d).

To further assess the therapeutic potential of amlexanox, we employed a remitting-relapsing EAE model, which involved the immunization of SJL mice with a peptide (139-151) derived from the proteolipid protein (PLP). As expected, the immunized SJL mice developed EAE, recovered from the disease after about 20 days and then relapsed (Fig. 6e). On day 23 after immunization, we treated the mice (via i.p. injection) with vehicle control or the TBK1 inhibitor amlexanox $(25 \mathrm{mg}$ per $\mathrm{kg}$ body weight) for 14 days $(n=5)$. Importantly, the amlexanox treatment greatly inhibited the EAE relapse (Fig. 6e). Collectively, these results highlight the potential of TBK1 as a therapeutic target for the treatment of MS. Of course, it is currently unclear whether the effect of amlexanox was solely on T cells or also on innate immune cells, such as monocytes or macrophages. Future studies will further address this question by generating myeloid cell-conditional Tbk1-KO mice.

\section{Discussion}

The data presented in this study establish TBK1 as a crucial signalling factor that regulates the homeostasis and inflammatory function of $\mathrm{T}$ cells. A prominent phenotype of the Tbk1-TKO mice was the increased frequency of memory-like $\mathrm{T}$ cells and decreased frequency of naïve $\mathrm{T}$ cells in the lymphoid organs. Although the TBK1 deficiency promotes T-cell activation and differentiation, the Tbkl-TKO mice are refractory to the induction of EAE, an animal model of the autoimmune neuroinflammatory disease MS.

We obtained genetic and biochemical evidences that TBK1 served as a negative regulator of AKT. T-cell-specific ablation of TBK1 caused heightened activation of AKT and its downstream targets, mTORC1 and Foxo1. The deregulated mTORC1 activation appeared to contribute to the perturbed homeostasis of TBK1-deficient T cells. We found that the injection of Tbk1-TKO mice with an mTORC1 inhibitor could largely, although not completely, correct their abnormal phenotype in T-cell 
a

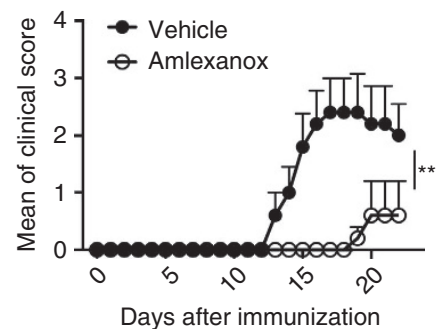

C

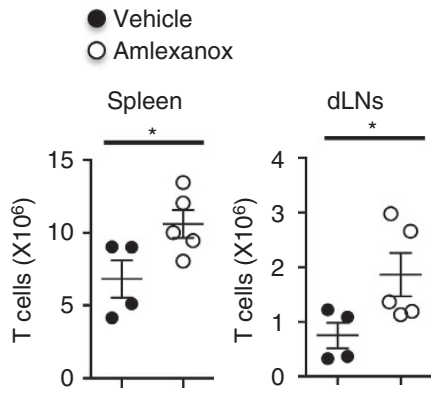

b

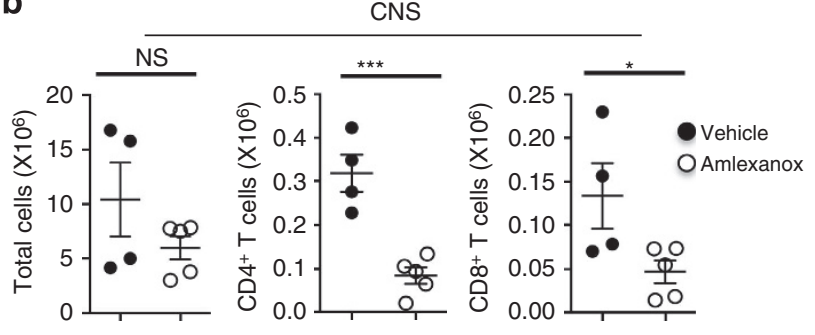

d

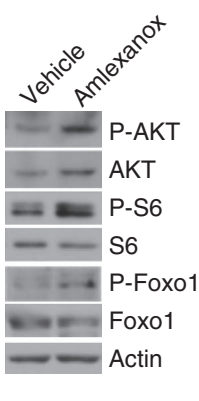

e

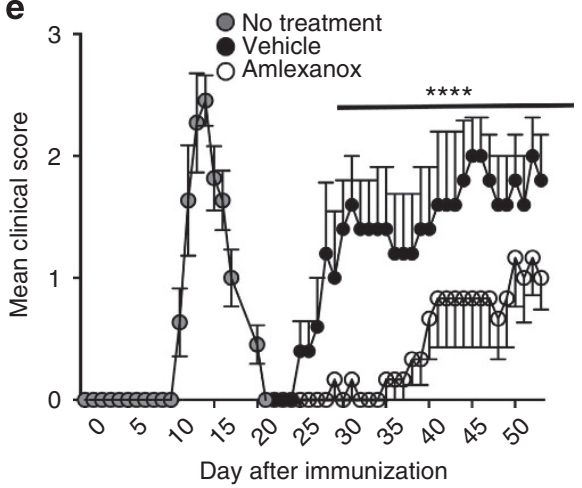

Figure 6 | A small-molecule inhibitor of TBK1 promotes AKT-mTORC1 activation and inhibits EAE induction. (a-d) WT mice were immunized for EAE induction. Starting from 4 days before the immunization, the mice were treated daily (via i.p. injection) with vehicle control or the TBK1 inhibitor amlexanox ( $25 \mathrm{mg}$ per $\mathrm{kg}$ body weight) for 14 days $(n=5)$. The immunized mice were monitored for EAE disease scores (a) or killed on day 22 post immunization for flow cytometric analysis of the indicated cell populations in the CNS (b) and peripheral lymphoid organs (c) or for IB analysis of the indicated phosphorylated (P-) and total proteins in the splenic CD4 ${ }^{+}$T cells (d). (e) EAE clinical score of SJL.J mice immunized with PLP $139-151$ to induce the remitting-relapsing EAE and then treated daily (via i.p. injection) with vehicle control or the TBK1 inhibitor amlexanox (25 mg per kg body weight) for 14 days starting from day $23(n=5)$. Data represent mean \pm s.d. from two or more independent experiments. ${ }^{\star} P<0.05$; ${ }^{* \star} P<0.01 ;{ }^{* \star \star} P<0.001$; ${ }^{\star \star \star \star \star} P<0.0001$; NS, non-significant as determined by two-way analysis of variance with Bonferroni's post test for EAE clinical scores analysis and two-tailed unpaired Student's $t$-test for other analysis, comparing the indicated groups.

homeostasis. These data suggest that the uncontrolled mTORC1 signalling contributes to, but may not be solely responsible for, the perturbed T-cell homeostasis in Tbk1-TKO mice. Besides mTORC1, several other factors in the related pathways, including the transcription factors Foxo1 and KLF2, are required for maintaining T-cell homeostasis ${ }^{37,38}$. Indeed, the TBK1 deficiency attenuated Foxo1 activation and KLF2 expression in T cells, indicating the involvement of these transcription factors in the perturbed T-cell homeostasis in the Tbk1-TKO mice.

Migration of autoimmune $\mathrm{T}$ cells from lymphoid organs to the target tissues represents a crucial and complex step in the development of autoimmunity. Newly activated $\mathrm{T}$ cells express low levels of the factors involved in T-cell migration, particularly the transcription factor KLF2 and its target gene product S1PR1, which is correlated with the lymphoid retention of the T cells ${ }^{39}$. Over time, the activated $\mathrm{T}$ cells differentiate into effector $\mathrm{T}$ cells and gradually regain the ability to express the migrationregulatory genes, which is important for the egress of $\mathrm{T}$ cells from the lymphoid organs. The role of KLF2 and S1PR1 in mediating T-cell migration has been directly demonstrated in a number of studies $37,40,41$. Our data suggest that TBK1 promotes the expression of KLF2 and S1PR1 in T cells and, thereby, facilitates the lymph node egress of $\mathrm{T}$ cells. We obtained genetic and pharmacological evidence that deregulated AKT activation in Tbk1-TKO T cells contributed to the reduced expression of KLF2 and S1PR1 and attenuated migration of $\mathrm{T}$ cells to the CNS. Ablation of one allele of $A k t$ in the Tbk1-TKO largely corrected their phenotype in T-cell migration and EAE sensitivity. The transcription factor Foxo1, which is negatively regulated by AKT, has been implicated in the induction of KLF2 and other migration-regulatory genes ${ }^{38,42,43}$. We propose that TBK1 promotes T-cell migration by downregulating AKT and, thereby, maintaining a sufficient level of active Foxol for mediating the expression of migration genes. Indeed, we found that TBK1 deficiency causes elevated Foxol phosphorylation in T cells.

It is important to note that the TBK1 deficiency only partially impaired the lymph node egress of $\mathrm{T}$ cells. Consistently, the Tbk1-TKO mice remained competent in mounting immune responses to influenza virus infection. This finding suggests that TBK1 may regulate additional functions of the CNS autoimmune $\mathrm{T}$ cells other than facilitating their exit from the draining lymph nodes. It is also possible that the complex route of autoimmune T-cell migration in EAE may contribute to the remarkable resistance of the Tbk1-TKO mice to EAE induction. Following their exit from draining lymph nodes, CNS autoimmune T cells migrate to the CNS via the lung and the lung-draining mediastinal lymph nodes ${ }^{24}$. Our data suggest that the Tbk1TKO effector T cells not only have the tendency to be retained in the draining lymph nodes but also accumulate in the mediastinal lymph nodes. These results suggest that the impaired CNS infiltration of TBK1-deficient T cells may involve different steps of lymph node retention, although it remains possible that TBK1 may also regulate additional mechanisms of T-cell migration to the CNS.

TBK1 and its homologue IKKe are known as an innate immune kinase that mediates induction of type I IFNs ${ }^{14}$. Although TBK1 is thought to be functionally redundant with IKK $\varepsilon$ in innate immune cells, our data suggest that these two kinases have fundamentally different functions in $\mathrm{T}$ cells. While 
TBK1 regulates T-cell homeostasis and mediates T-cell migration into the CNS, IKKE is dispensable for these biological functions. IKKe is also dispensable for the control of AKT-mTORC1 signalling axis. Nevertheless, like TBK1, IKKe is activated by the $\mathrm{T}$-cell activation signals. Further studies are required to examine whether IKKE regulates any aspects of T-cell functions. The functional differences between TBK1 and IKK $\varepsilon$ have also been shown in B cells, in which TBK1, but not IKKe, regulates antibody class switching to $\operatorname{IgA}{ }^{44}$.

Studies using immortalized mouse embryonic fibroblast and cancer cell lines suggest a positive role of TBK1 in the regulation of $\mathrm{AKT}^{29,45}$. TBK1 phosphorylates AKT at several residues, including threonine-195 and serine-378, although the functional significance has not been defined ${ }^{29}$. Our current work provides the first evidence for the in vivo role of TBK1 in the regulation of AKT. Our data clearly demonstrated a negative role for TBK1 in AKT regulation. TBK1 does not seem to inhibit the catalytic activation, but rather controls the fate, of AKT. In TBK1-deficient $\mathrm{T}$ cells, the steady level of AKT is drastically elevated, which contributes to the aberrant AKT activity in naïve and effector $\mathrm{T}$ cells. We have obtained biochemical evidence that the serine378 phosphorylation site of AKT is crucial for TBK1-stimulated AKT degradation.

Our work not only demonstrated a novel signalling mechanism that regulates T-cell homeostasis and EAE pathogenesis but also have profound implications for MS therapy. Regarding the human relevance of our findings, we found that TBK1 knockdown in human PBMCs causes hyperactivation of AKTmTORC1 signalling and reduced expression of the T-cell homing genes KFL2 and S1PR1. Furthermore, TBK1 knockdown or pharmacological inhibition attenuates the transmigration of human $\mathrm{CD}^{+} \mathrm{T}$ cells across a human brain microvascular endothelial monolayer in vitro. Our finding that TBK1 ablation does not affect $\mathrm{T}$-cell activation or immune responses against flu viral infection, but attenuates EAE induction, makes TBK1 an attractive therapeutic target to be exploited in the treatment of MS. We found that amalexanox, a Food and Drug Administration-approved drug that selectively inhibits TBK1 (ref. 36), greatly attenuated the induction of EAE and inhibited the disease relapse in a remitting-relapsing model of EAE. Amlexanox also inhibited human T-cell migration in vitro. Of note, the mode of action of TBK1 differs substantially from that of S1PR1. First of all, TBK1 appears to regulate the re-expression of S1PR1 following its downregulation in activated $\mathrm{T}$ cells, but is dispensable for the overall expression of S1PR1. Thus, unlike the S1PR1 ablation, the TBK1 deficiency does not influence the homing of naïve $T$ cells or the thymic emigration of newly generated T cells. Furthermore, the TBK1 deficiency only partially inhibits the expression of S1PR1 and the egress of effector $T$ cells from the lymph nodes. Consistently, the loss of TBK1 does not appreciably compromise immune responses against influenza virus infection. These findings along with the fact that amlexanox is a relatively safe drug that has been used for many years in the clinic indicate that amlexanox may be an attractive compound to be evaluated in MS clinical trials.

\footnotetext{
Methods

Mice. The Tbk1-flox mice were generated in $\mathrm{B} 6 \times 129$ mixed genetic backgrounds $^{44}$ and further backcrossed to the $\mathrm{B} 6$ mice for four generations. To generate T-cell-conditional Tbk1-KO mice, Tbk1-flox mice ${ }^{44}$ were crossed with CD4-Cre transgenic mice (B6 genetic background, Jackson Laboratories), producing Tbk1 ${ }^{+1+} \mathrm{CD} 4-\mathrm{Cre}$ (named WT) and Tbk1 $1^{\mathrm{fl} / \mathrm{l}} \mathrm{CD} 4$-Cre (named Tbk1-TKO mice) mice. Tbk1-flox mice were also crossed with Rosa26-Cre-ER mice (B6 genetic background, Jackson Laboratories) and the progeny Tbk1 $+1+$ Rosa26-Cre-ER and $T b k 1^{\mathrm{l} / \mathrm{fl}}$ Rosa26-Cre-ER mice were injected i.p. with tamoxifen ( $2 \mathrm{mg}$ per mouse) in corn oil daily for four consecutive days to induce Cre function. The tamoxifentreated $T b k 1^{+} I+$ Rosa26-Cre-ER and $T b k 1^{\mathrm{fl} / \mathrm{fl}}$ Rosa26-Cre-ER mice were named
}

WT-ER and Tbk1-ER mice, respectively, and used 2 weeks after the treatment. Carma1-KO mice (B6 $\times 129$ genetic background) were provided by Dr Josef $\mathrm{M}$. Penninger (Austrian Academy of Sciences) ${ }^{46}$. Mice with loxP-flanked allele encoding IKK $\beta$ (IKK2) or NEMO alleles were provided by Dr Manolis Pasparakis (University of Cologne) ${ }^{47}$. These mice were crossed with CD4-Cre mice to generate the T-cell-conditional IKK $\beta$ KO (IKK $\beta$-TKO) and NEMO KO (NEMO-TKO) mice. IKK $\varepsilon-\mathrm{KO}$, B6.SJL (CD45.1 ${ }^{+}$), C57BL/6, Rag1-KO and TCR $\beta / \delta$ KO mice were from Jackson Laboratory. Experiments were performed with age-matched mice. Mice were maintained in a specific pathogen-free facility of The University of Texas MD Anderson Cancer Center, and all animal experiments were done in accordance with protocols approved by the Institutional Animal Care and Use Committee of the University of Texas MD Anderson Cancer Center.

Cell lines. The NEMO-deficient JM4.5.2 cell line and its NEMO-competent control J.SVT35 are Jurkat derivatives ${ }^{18}$. The CARMA1-deficient JPM50.6 mutant cell line and its parental Jurkat cell line were provided by Dr Xin Lin (UT MD Anderson Cancer Center) ${ }^{17}$.

Plasmids and antibodies and reagents. The pcDNA expression vector encoding haemagglutinin (HA)-tagged human AKT1 was provided by Dr Keqiang Ye (Emory University). pMIGR1-hAKT was generated by inserting human AKT1 cDNA into the EcoRI and BglII sites of pMIGR1 vector, and the AKT mutants (T195A, T 308A, S378A) were created by site-directed mutagenesis. Flag-tagged TBK1 and its catalytically inactive mutant, K38A, were provided by Dr Chen Wang

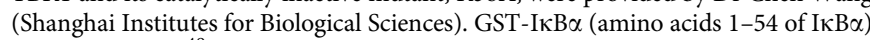
was as described ${ }^{48}$ and GST-IRF3 (amino acids 380-427) was provided by Dr Rongtuan Lin (McGill University).

Functional grade anti-mouse (m) CD3e (145-2C11) and anti-mCD28 (37.51) antibodies and blocking antibodies for mIFN- $\gamma$ (XMG1.2) and mIL-4 (11B11) were from eBioscience. Antibodies for AKT1 (B-1, 1:1,000), phospho-ERK1/2 (E-4, 1:1,000), ERK1/2 (K-23, 1:1,000), p38 (H-147, 1:1,000), and GFP (B-2, 1:1,000) were from Santa Cruz Biotechnology. Anti-IKKi (IKKe, 1:1,000), anti-Actin (C-4, 1:5,000) and horseradish peroxidase-conjugated anti-Flag (M2, 1:10,000) were from Sigma-Aldrich. Antibodies for phospho-TBK1 Ser172 (D52C2, 1:1,000), phospho-AKT1 Ser473 (D9E, 1:1,000), phospho-S6K1 Thr389 (1A5, 1:1,000), phospho-S6 Ser235/236 (D57.2.2E, 1:1,000), phospho-FoxO1 Thr24/FoxO3a Thr32 (1:1,000), phospho-p38 Thr180/Tyr182 (3D7, 1:1,000), TBK1, S6K1 (49D7, 1:1,000), S6 (54D2, 1:1,000) and FoxO1 (C29H4, 1:1,000) were from Cell Signaling Technology. Horseradish peroxidase-conjugated anti-haemagglutinin (HA-7, 1:3,000) was from Roche. Anti-K48-Ubiquitin (Apu2, 1:1,000) was from Millipore.

Fluorescence-labelled antibodies for murine (m) CD4 (L3T4, 1:300), mCD8 (53-6.7, 1:300), mCD3 (145-2C11, 1:300), CD44 (IM7, 1:300), CD62L (MEL-14, 1:300), mTCR $\beta$ (H57-597, 1:300), mCD45.1 (A20, 1:300), mCD45.2 (104, 1:300), mThy1.1 (HIS51, 1:300), IL-17A (eBio17B7, 1:300), and IFN- $\gamma$ (XMG1.2, 1:300) were purchased from eBioscience. Annexin V was from BD. The fluorescencelabelled phospho-specific antibodies were all from Cell Signaling Technology.

The AKT inhibitor $1 / 2$ was from Calbiochem, the PI3 kinase inhibitor LY294002 was from Selleck, the TBK1 inhibitor MRT673037 was from Chemexpress, the TBK1 inhibitor amlexanox was from Cayman Chemical and the protein synthesis inhibitor cycloheximide was from Sigma-Aldrich.

Flow cytometry analysis and cell sorting. Single-cell suspensions of splenocytes and lymph node cells were subjected to flow cytometry and cell sorting as previously described ${ }^{49}$ using a FACSAria (BD Biosciences). For intracellular cytokine staining (ICS) assays, T cells isolated from the spleen, draining lymph nodes or CNS (brain and spinal cord) of immunized mice or from in vitro cultures were stimulated for $4 \mathrm{~h}$ with PMA (50 $\left.\mathrm{ng} \mathrm{ml}^{-1}\right)$ and Ionomycin $\left(500 \mathrm{ng} \mathrm{ml}^{-1}\right)$ in the presence of monensin $\left(10 \mu \mathrm{g} \mathrm{ml}^{-1}\right)$. The stimulated cells were fixed in $2 \%$ paraformaldehyde and permeablized in $0.5 \%$ saponin and then subjected to cytokine staining flow cytometry analyses.

In vivo inhibition of mTORC1. Mice were injected daily (i.p) with the mTORC1 inhibitor rapamycin ( $3 \mathrm{mg}$ per $\mathrm{kg}$ body weight) or solvent control ( $1 \%$ ethanol in PBS) for 5 days and then killed for experiments.

Influenza viral infection. Influenza virus A/PR8 (H1N1) was provided by Dr Brian E. Gilbert (Baylor College of Medicine). For influenza infection, mice were first anaesthetized and then inoculated intranasally with $50 \mu \mathrm{l}$ of influenza virus A/PR8 diluted in DMEM medium. Control mice were inoculated with $50 \mu \mathrm{L}$ DMEM as control. Body weight was monitored every other day for 8 days. On day 8 , bronchoalveolar lavage fluid was collected in anaesthetized mice. Then, $500 \mu \mathrm{l}$ cold PBS was instilled into the lungs through the trachea and aspirated back. After collecting bronchoalveolar lavage fluid, mice were killed for cell distribution analysis.

Induction and evaluation of EAE. The encephalitogenic peptide of MOG (residues 35-55, Met-Glu-Val-Gly-Trp-Tyr-Arg-Ser-Pro-Phe-Ser-Arg-Val-Val-HisLeu-Tyr-Arg-Asn-Gly-Lys) and the encephalitogenic peptide of myelin PLP 
(residues 139-151, His-Cys-Leu-Gly-Lys-Trp-Leu-His-Pro-Asp-Lys-Phe) were purchased from Genemed Synthesis Inc. (San Francisco, CA, 95\% purity). To induce acute EAE, mice were injected subcutaneously (in the back region) with $200 \mu \mathrm{g}$ of the $\mathrm{MOG}_{35-55}$ peptide in CFA containing $5 \mathrm{mg} \mathrm{ml}^{-1}$ heat-killed $\mathrm{Myco}$ bacterium tuberculosis (H37Ra strain; BD Diagnostics, Franklin Lakes, NJ). To induce relapse EAE, SJL.J mice were immunized with $150 \mu \mathrm{g}$ of $\mathrm{PLP}_{139-151}$ in CFA containing $5 \mathrm{mg} \mathrm{ml}^{-1}$ heat-killed Mycobacterium tuberculosis. For both types of induction, on the day of immunization and $48 \mathrm{~h}$ later, the mice were also injected i.v. with Pertussis toxin (200 ng per mouse; List Biological Laboratories, Campbell, CA) in PBS. Mice were examined daily for EAE disease symptoms, which were scored using a standard method: 0 , no clinical signs; 1 , limp tail; 2 , paraparesis (weakness, incomplete paralysis of one or two hind limbs); 3, paraplegia (complete paralysis of two hind limbs); 4, paraplegia with fore limb weakness or paralysis; and 5 , moribund state or death. The EAE scores were assessed without group allocation information.

For amlexanox treatment, mice were i.p. injected with amlexanox $(25 \mathrm{mg}$ per $\mathrm{kg}$ body weight) or vehicle (dimethylsulphoxide $50 \%$ and corn oil $50 \%$ ) control daily for total of 14 days. Mice were randomly selected for receiving amlexanox treatment.

For passive EAE, WT and Tbk1-TKO mice were immunized with $\mathrm{MOG}_{35-55}$ peptide for 10 days. Draining $\mathrm{LN}$ and spleen cells were then isolated and in vitro expanded for $72 \mathrm{~h}$ in the presence of $30 \mu \mathrm{g} \mathrm{ml}^{-1} \mathrm{MOG}_{35-55}$ peptide and Th17differentiation agents $\left(10 \mu \mathrm{g} \mathrm{ml}^{-1}\right.$ anti-IFN $\gamma, 10 \mathrm{ng} \mathrm{ml}^{-1}$ IL-1 $\beta$ and $10 \mathrm{ng} \mathrm{ml}^{-1}$ IL-23). $\mathrm{CD} 4^{+} \mathrm{T}$ cells were purified and adoptively transferred into irradiated (500 rads) recipient mice to for passive EAE induction.

Analysis of in vivo T-cell differentiation and CNS infiltration. At the indicated times after $\mathrm{MOG}_{35-55}$ immunization, the mice were killed for splenocyte preparation and CNS infiltration analysis. $\mathrm{CD} 4{ }^{+} \mathrm{T}$ cells were isolated from the splenocytes using magnetic beads (Invitrogen) and subjected to ICS as described above. For the preparation of CNS lymphocytes, brains and spinal cords were excised and dissociated for $1 \mathrm{~h}$ at $37^{\circ} \mathrm{C}$ by digestion with collagenase IV $\left(0.5 \mathrm{mg} \mathrm{ml}^{-1}\right.$; Invitrogen) and DNase I $\left(10 \mu \mathrm{g} \mathrm{ml}^{-1}\right.$; Roche, Indianapolis, IN) in RPMI medium. Dispersed cells were passed through a $40-\mu \mathrm{m}$ nylon mesh and collected by centrifugation. The cells were then resuspended in RPMI medium, layered onto a Percoll density gradient (Biochrom, Berlin, Germany) and centrifuged for $30 \mathrm{~min}\left(625 \mathrm{~g}, 22^{\circ} \mathrm{C}\right)$. CNS lymphocytes were isolated by collection of the interphase fraction between 30 and $70 \%$ Percoll. After intensive washing in Hanks balanced-salt solution, cells were analysed by flow cytometry.

Histology and immunohistochemistry. We dissected spinal cords from mice transcardially perfused with $4 \%(\mathrm{w} / \mathrm{v})$ paraformaldehyde and postfixed them overnight. We then stained the paraffin-embedded sections $(8 \mu \mathrm{m})$ of spinal cords with haematoxylin and eosin or Luxol Fast Blue staining. Cryostat sections (7-8 $\mu \mathrm{m})$ of lymph nodes were fixed and stained with biotinylated anti-CD4 (1:100; BD Biosciences) at $4{ }^{\circ} \mathrm{C}$ overnight, followed by HRP-conjugated streptavidin (Thermo Scientific) at room temperature for $1 \mathrm{~h}$. Slices were then developed with peroxidase substrate kit (Vector)

Mixed bone marrow chimera. Rag1-KO mice were subjected to lethal-dose (992 rads) irradiation and, 1 day later, were adoptively transferred with bone marrow cells harvested from the tibiae and femurs of the indicated mice. For mixed bone marrow transfers, an equal number $\left(6.4 \times 10^{6}\right)$ of bone marrows from the CD45.1 $1^{+}$B6.SJL and the CD45.2 ${ }^{+}$WT or Tbk1-TKO mice or from the GFP ${ }^{+}$ WT and the GFP ${ }^{-}$Tbk1-TKO mice were mixed and injected i.v. into the irradiated Rag1-KO mice. After 6 weeks, the resulting chimeras were either directly analysed or subjected to EAE induction.

Adoptive transfer of T cells for homing studies. WT and Tbk1-TKO T cells were incubated at $37^{\circ} \mathrm{C}$ for $15 \mathrm{~min}$ with $1 \mathrm{mM}$ CFSE and $5 \mathrm{mM}$ CMRA (Molecular Probes), respectively, according to manufacturer's protocol. The differentially labelled WT and Tbk1-TKO T-cell preparations were mixed at 1:1 ratio $\left(5 \times 10^{6}\right.$ cells each) and injected i.v. into the indicated recipient mice, followed by immunizing the mice with $\mathrm{MOG}_{35-55}$ peptide. Lymphatic vessels and the draining inguinal lymph nodes were labelled in vivo by injecting mice (subcutaneous) with $5 \mu \mathrm{g}$ of anti-mouse LYVE-1-AF647 antibody in $100 \mu \mathrm{l}$ PBS in the flank $24 \mathrm{~h}$ before imaging. For static imaging, inguinal LNs were excised, fixed in 3\% paraformaldehyde PBS and cut in half. Each half lymph node was whole-mounted on a cover slip imaged using Leica SP5 RS AOBS confocal microscope with a $\times 20$ NA0.75 objective and silicon immersion. Three-dimensional image stacks were acquired with $4 \mu \mathrm{m} z$-spacing. Three-dimensional analysis was performed using Imaris 7.7 software.

T-cell purification and in vitro treatments. $\mathrm{CD} 4^{+} \mathrm{T}$ cells were purified from splenocytes with anti-CD4-conjugated magnetic beads (Invitrogen). Inhibitor studies were carried out by culturing the cells in 24 -well plates $\left(4 \times 10^{6}\right.$ cells per well) in the presence of AKT1/2 inhibitor (AKTi, $1 \mu \mathrm{M})$, the PI3 kinase inhibitor $(\mathrm{LY} 294002,50 \mu \mathrm{M})$ or the TBK1 inhibitor (MRT67307, $2 \mu \mathrm{M})$. For ELISA and proliferation assays, naïve $\mathrm{CD} 4 \mathrm{~T}$ cells were stimulated in replicate wells of 96-well plates $\left(1 \times 10^{5}\right.$ cells per well). Culture supernatants were analysed by ELISA (eBioScience), and cells were pulse-labelled for $6 \mathrm{~h}$ with $\left[{ }^{3} \mathrm{H}\right]$ thymidine for proliferation assays.

For in vitro $\mathrm{CD} 4{ }^{+} \mathrm{T}$-cell differentiation assays, naïve $\mathrm{CD} 4{ }^{+} \mathrm{T}$ cells $\left(\mathrm{CD} 4{ }^{+} \mathrm{CD} 25^{-} \mathrm{CD} 44^{\mathrm{lo}} \mathrm{CD} 62 \mathrm{~L}^{\text {hi }}\right)$ were sorted from splenic CD4 ${ }^{+} \mathrm{T}$ cells, prepared using a CD4 T-cell Isolation Kit (Miltenyi Biotec, Auburn, CA) and stimulated with plate-bound anti-CD3 and anti-CD28 under Th0 $\left(5 \mu \mathrm{g} \mathrm{ml}{ }^{-1}\right.$ anti-IL-4, $5 \mu \mathrm{g} \mathrm{ml}^{-1}$ anti-IFN- $\gamma$ ), Th1 (10 $\mathrm{ng} \mathrm{ml}^{-1}$ IFN- $\gamma, 10 \mathrm{ng} \mathrm{ml}^{-1} \mathrm{IL}-12,5 \mu \mathrm{g} \mathrm{ml}^{-1}$ anti-IL-4) or Th17 (20 ng ml ${ }^{-1}$ IL-6, $5 \mathrm{ng} \mathrm{ml}^{-1}$ TGF- $\beta, 5 \mu \mathrm{g} \mathrm{ml}^{-1}$ anti-IL-4, $5 \mu \mathrm{g} \mathrm{ml}^{-1}$ anti-IFN- $\gamma$ ) conditions. After the indicated times, the cells were subjected to ICS to quantify the production of their signature cytokines.

Retroviral and lentiviral infections. Retroviral particles were prepared using the indicated pMIGR1-GFP-based expression vectors, as previously described ${ }^{50}$. For the production of lentiviral particles, HEK293T cells were transfected (by calcium method) with pLKO.1 lentiviral vectors encoding shRNAs for either the control luciferase protein or TBK1 along with the packaging vectors psPAX2 and pMD2. Naive $\mathrm{CD} 4{ }^{+} \mathrm{T}$ cells were activated with plate-bound anti-CD3 $\left(1 \mu \mathrm{g} \mathrm{ml}^{-1}\right)$ plus anti-CD28 $\left(1 \mu \mathrm{g} \mathrm{ml}^{-1}\right)$ in 24 -well plates for $36 \mathrm{~h}$ and then infected with retroviruses. After $48 \mathrm{~h}$, the transduced cells were either enriched by flow cytometric cell sorting (based on GFP expression) for in vitro assays or directly transferred into T-cell-deficient mice for in vivo assays.

PBMCs were isolated from human whole blood samples (purchased from The Gulf Coast Regional Blood Center) by Ficoll density gradient centrifugation. The cells were activated for $24 \mathrm{~h}$ with anti-CD3 plus anti-CD28 and then infected with the control or TBK shRNA lentiviral particles. Following infection, the infected cells were selected by culturing the cells in the presence of puromycin for $48 \mathrm{~h}$. The use of human blood samples was approved by the Institutional Review Board for Human Research at MD Anderson Cancer Center.

In vitro human T-cell migration assays. In vitro $\mathrm{T}$-cell migration assays were performed using a 24 -well plate Boyden chamber essentially as previously described $^{33}$. We seeded $1 \times 10^{5}$ primary human brain microvascular ECs (ACBRI 376) on top of a $3-\mu \mathrm{m}$ pore size membrane in endothelial cell culture media. After 5 days of cultivation, the cells formed a confluent monolayer. At that point, the ECs were treated for $24 \mathrm{~h}$ with $100 \mathrm{ng} \mathrm{ml}^{-1}$ of recombinant human IL-17 (R\&D Systems). The next day, ECs were washed and a suspension of $1 \times 10^{6} \mathrm{CD} 4$ human $\mathrm{T}$ cells was loaded in the upper chamber. The migration ability of $\mathrm{T}$ cells was assessed by counting the absolute number of $\mathrm{T}$ cells that transmigrated to the lower chamber after $18 \mathrm{~h}$

Immunoblot and kinase assays. For protein phosphorylation analysis and kinase assays, purified $\mathrm{T}$ cells were stimulated by a crosslinking method ${ }^{49}$. In brief, the cells were incubated on ice with anti-CD3 $\left(2 \mu \mathrm{g} \mathrm{ml}{ }^{-1}\right)$ and anti-CD28 $\left(2 \mu \mathrm{g} \mathrm{ml}^{-1}\right)$, followed by crosslinking with goat anti-hamster Ig $\left(25 \mu \mathrm{g} \mathrm{ml}^{-1}\right)$ for different time periods at $37^{\circ} \mathrm{C}$ and then immediately lysed in a kinase cell lysis buffer supplemented with phosphatase inhibitors. For IB analyses using transiently transfected cells, HEK293T cells were transfected using calcium method and cultured for $48 \mathrm{~h}$. The cells were either treated as indicated or directly collected for lysate preparation. IB analyses were performed using antibodies that detect phosphorylated AKT (Ser473, 1:1,000), Foxo1 (Thr24, 1:1,000), S6 (Ser235/236, 1:1,000), S6K1 (Thr389, 1:1,000), p38 (Thr180/Tyr182, 1:1,000) and ERK1/2 (Tyr204, 1:1,000), or their total protein controls. In vitro kinase assays were as described ${ }^{51}$.

For ubiquitination assays, thymocytes were isolated from young (6 weeks old) WT or Tbkl-TKO mice and immediately lysed in RIPA buffer $(50 \mathrm{mM}$ Tris- $\mathrm{HCl}$ $\mathrm{pH} 7.4,150 \mathrm{mM} \mathrm{NaCl}, 1 \%(\mathrm{v} / \mathrm{v})$ Nonidet P-40, $0.5 \%(\mathrm{v} / \mathrm{v})$ sodium deoxycholate and $1 \mathrm{mM}$ EDTA) supplemented with $4 \mathrm{mM} \mathrm{N}$-ethylmaleimide. Lysates were immediately boiled for $5 \mathrm{~min}$ in the presence of $1 \%$ SDS and then diluted 10 times with RIPA buffer. Ubiquitinated AKT was isolated by immunoprecipitation using anti-AKT and detected by IB using an anti-ubiquitin antibody that specifically recognizes K48 ubiquitin chains.

Full size images are presented in Supplementary Figs 8-11.

Real-time quantitative PCR. RNA was extracted with TRIzol reagent (Sigma) from either mouse T cells or human PBMCs derived from healthy donors or relapsing-remitting MS patients. Specimen collection was approved by the Committee for the Protection of Human Subjects of the University of Texas Health Science Center at Houston, and informed consent was obtained from all subjects. The RNA samples were subjected to quantitative PCR analyses using the SYBR regent (Bio-Rad). The expression of individual genes was calculated by a standard curve method and was normalized to the expression of Actb. The gene-specific primer sets used (all for mouse genes) were as follows: CCR7, 5'-GCACCATGGA CCCAGGGAAACC- $3^{\prime}$ and $5^{\prime}$-AGTCATCGGTGACCTCATCTTGGCA-3'; KLF2, $5^{\prime}$-TATCTTGCCGTCCTTTGCCA-3' and $5^{\prime}$-TTTAGGTCCTCATCCGTGCC-3'; CD62L, $5^{\prime}$-AACGATGACGCCTGTCACAA- $3^{\prime}$ and $5^{\prime}$-GTTTCCACACATTCT CCACGG-3'; S1prl, 5'-GGTGTAGACCCAGAGTCCTGC-3' and $5^{\prime}$-AGAGGCC TCCGAGAAACAGC-3'; AKT1, 5'-AAGAAGGAGGTCATCGTCGC- $3^{\prime}$ and 5'-CTTGAGGGCCGTAAGGAAGG-3'; Itga4, 5' -AAGCATCCCTGGCCACTA 
C- $3^{\prime}$ and $5^{\prime}$-GAGCGCCCCAAGAGATGAAG- ${ }^{\prime}$; Itgb1, $5^{\prime}$-AACTTGTTGGTCAG CAACGC- $3^{\prime}$ and $5^{\prime}$-AGCCAATCAGCGATCCACAA- ${ }^{\prime}$; Ninj1, $5^{\prime}$-AGTCGGGC ACTGAGGAGTAT- $3^{\prime}$ and $5^{\prime}$-TACATTGATGGGCCGGTTCC- ${ }^{\prime}$.

Statistical analysis. For the EAE clinical scores, differences between groups were evaluated by two-way analysis of variance with Bonferroni's post test. Other statistical analyses were performed by two-tailed unpaired $t$-test using the Prism software. $P$ values less than 0.05 were considered significant and the level of significance was indicated as ${ }^{*} P<0.05,{ }^{* *} P<0.01,{ }^{* *} P<0.001$ and ${ }^{* * *} P<0.0001$.

In our animal studies, three to four mice are required for each group based on our calculation to achieve a 2.3 -fold change (effect size) in two-tailed $t$-test with $90 \%$ power and a significance level of 5\%. All statistical tests justified as appropriate and the data meet the assumptions of the tests. The variance is similar between the groups that are being statistically compared.

\section{References}

1. Ohashi, P. S. T-cell signalling and autoimmunity: molecular mechanisms of disease. Nat. Rev. Immunol. 2, 427-438 (2002).

2. Frohman, E. M., Racke, M. K. \& Raine, C. S. Multiple sclerosis-the plaque and its pathogenesis. N. Engl. J. Med. 354, 942-955 (2006).

3. Simmons, S. B., Pierson, E. R., Lee, S. Y. \& Goverman, J. M. Modeling the heterogeneity of multiple sclerosis in animals. Trends Immunol. 34, 410-422 (2013).

4. Goverman, J. Autoimmune T cell responses in the central nervous system. Nat. Rev. Immunol. 9, 393-407 (2009).

5. Sallusto, F. et al. T-cell trafficking in the central nervous system. Immunol. Rev. 248, 216-227 (2012).

6. Zhu, J., Yamane, H. \& Paul, W. E. Differentiation of effector CD4 T cell populations. Annu. Rev. Immunol. 28, 445-489 (2010).

7. Fitzgerald, K. A. et al. IKKepsilon and TBK1 are essential components of the IRF3 signaling pathway. Nat. Immunol. 4, 491-496 (2003).

8. Sharma, S. et al. Triggering the interferon antiviral response through an IKK-related pathway. Science 300, 1148-1151 (2003).

9. McWhirter, S. M. et al. IFN-regulatory factor 3-dependent gene expression is defective in Tbk1-deficient mouse embryonic fibroblasts. Proc. Natl Acad. Sci. USA 101, 233-238 (2004).

10. Hemmi, H. et al. The roles of two IkappaB kinase-related kinases in lipopolysaccharide and double stranded RNA signaling and viral infection. J. Exp. Med. 199, 1641-1650 (2004).

11. Perry, A. K., Chow, E. K., Goodnough, J. B., Yeh, W. C. \& Cheng, G. Differential requirement for TANK-binding kinase-1 in type I interferon responses to toll-like receptor activation and viral infection. J. Exp. Med. 199, 1651-1658 (2004).

12. Solt, L. A. \& May, M. J. The IkappaB kinase complex: master regulator of NF-kappaB signaling. Immunol. Res. 42, 3-18 (2008).

13. Verhelst, K., Verstrepen, L., Carpentier, I. \& Beyaert, R. IkappaB kinase epsilon (IKKepsilon): a therapeutic target in inflammation and cancer. Biochem. Pharmacol. 85, 873-880 (2013).

14. Hiscott, J. Convergence of the NF-kappaB and IRF pathways in the regulation of the innate antiviral response. Cytokine Growth Factor Rev. 18, 483-490 (2007).

15. Bonnard, M. et al. Deficiency of T2K leads to apoptotic liver degeneration and impaired NF-KB-dependent gene transcription. EMBO J. 19, 4976-4985 (2000).

16. Gaide, O. et al. CARMA1 is a critical lipid raft-associated regulator of TCRinduced NF-kappa B activation. Nat. Immunol. 3, 836-843 (2002).

17. Wang, D. et al. A requirement for CARMA1 in TCR-induced NF-kappa B activation. Nat. Immunol. 3, 830-835 (2002).

18. Harhaj, E. W. et al. Somatic mutagenesis studies of NF-kappa B signaling in human $\mathrm{T}$ cells: evidence for an essential role of IKK gamma in NF-kappa B activation by T-cell costimulatory signals and HTLV-I Tax protein. Oncogene 19, 1386-1391 (2000).

19. Harp, J. R. \& Onami, T. M. Naive T cells re-distribute to the lungs of selectin ligand deficient mice. PLoS ONE 5, e10973 (2010).

20. Arbones, M. L. et al. Lymphocyte homing and leukocyte rolling and migration are impaired in L-selectin-deficient mice. Immunity 1, 247-260 (1994).

21. Finlay, D. \& Cantrell, D. A. Metabolism, migration and memory in cytotoxic T cells. Nat. Rev. Immunol. 11, 109-117 (2011).

22. Reboldi, A. et al. C-C chemokine receptor 6-regulated entry of TH-17 cells into the CNS through the choroid plexus is required for the initiation of EAE. Nat. Immunol. 10, 514-523 (2009).

23. Sporici, R. \& Issekutz, T. B. CXCR3 blockade inhibits T-cell migration into the CNS during EAE and prevents development of adoptively transferred, but not actively induced, disease. Eur. J. Immunol. 40, 2751-2761 (2010).

24. Odoardi, F. et al. T cells become licensed in the lung to enter the central nervous system. Nature 488, 675-679 (2012).

25. Fabre, S. et al. Stable activation of phosphatidylinositol 3-kinase in the $\mathrm{T}$ cell immunological synapse stimulates Akt signaling to FoxO1 nuclear exclusion and cell growth control. J. Immunol. 174, 4161-4171 (2005).
26. Chi, H. Regulation and function of mTOR signalling in T cell fate decisions. Nat. Rev. Immunol. 12, 325-338 (2012).

27. Sprent, J. \& Surh, C. D. Normal T cell homeostasis: the conversion of naive cells into memory-phenotype cells. Nat. Immunol. 12, 478-484 (2011).

28. Clark, K. et al. Novel cross-talk within the IKK family controls innate immunity. Biochem. J. 434, 93-104 (2011).

29. Joung, S. M. et al. Akt contributes to activation of the TRIF-dependent signaling pathways of TLRs by interacting with TANK-binding kinase 1 . J. Immunol. 186, 499-507 (2011).

30. Pelletier, D. \& Hafler, D. A. Fingolimod for multiple sclerosis. N. Engl. J. Med. 366, 339-347 (2012)

31. Groves, A., Kihara, Y. \& Chun, J. Fingolimod: direct CNS effects of sphingosine 1-phosphate (S1P) receptor modulation and implications in multiple sclerosis therapy. J. Neurol. Sci. 328, 9-18 (2013).

32. Finlay, D. \& Cantrell, D. Phosphoinositide 3-kinase and the mammalian target of rapamycin pathways control T cell migration. Ann. NY Acad. Sci. 1183, 149-157 (2010).

33. Kebir, H. et al. Human TH17 lymphocytes promote blood-brain barrier disruption and central nervous system inflammation. Nat. Med. 13, 1173-1175 (2007).

34. Zhang, F., Shi, Y., Wang, L. \& Sriram, S. Role of HDAC3 on p53 expression and apoptosis in T cells of patients with multiple sclerosis. PLoS ONE 6, e16795 (2011).

35. Kemppinen, A. K., Kaprio, J., Palotie, A. \& Saarela, J. Systematic review of genome-wide expression studies in multiple sclerosis. BMJ Open 1, e000053 (2011).

36. Reilly, S. M. et al. An inhibitor of the protein kinases TBK1 and IKK-varepsilon improves obesity-related metabolic dysfunctions in mice. Nat. Med. 19, 313-321 (2013).

37. Sebzda, E., Zou, Z., Lee, J. S., Wang, T. \& Kahn, M. L. Transcription factor KLF2 regulates the migration of naive $T$ cells by restricting chemokine receptor expression patterns. Nat. Immunol. 9, 292-300 (2008).

38. Kerdiles, Y. M. et al. Foxol links homing and survival of naive T cells by regulating L-selectin, CCR7 and interleukin 7 receptor. Nat. Immunol. 10, 176-184 (2009).

39. Masopust, D. \& Schenkel, J. M. The integration of T cell migration, differentiation and function. Nat. Rev. Immunol. 13, 309-320 (2013).

40. Pham, T. H., Okada, T., Matloubian, M., Lo, C. G. \& Cyster, J. G. S1P1 receptor signaling overrides retention mediated by $\mathrm{G}$ alpha $\mathrm{i}$-coupled receptors to promote $\mathrm{T}$ cell egress. Immunity 28, 122-133 (2008).

41. Carlson, C. M. et al. Kruppel-like factor 2 regulates thymocyte and T-cell migration. Nature 442, 299-302 (2006).

42. Fabre, S. et al. FOXO1 regulates L-Selectin and a network of human T cell homing molecules downstream of phosphatidylinositol 3-kinase. J. Immunol. 181, 2980-2989 (2008).

43. Rougerie, P. et al. Fam65b is a new transcriptional target of FOXO1 that regulates RhoA signaling for T lymphocyte migration. J. Immunol. 190, 748-755 (2013).

44. Jin, J. et al. The kinase TBK1 controls IgA class switching by negatively regulating noncanonical NF-kappaB signaling. Nat. Immunol. 13, 1101-1109 (2012).

45. Xie, X. et al. I\{kappa\}B kinase \{varepsilon\} and TANK-binding kinase 1 activate AKT by direct phosphorylation. Proc. Natl Acad. Sci. USA 108, 6474-6479 (2011).

46. Hara, H. et al. The MAGUK family protein CARD11 is essential for lymphocyte activation. Immunity 18, 763-775 (2003).

47. Pasparakis, M. et al. TNF-mediated inflammatory skin disease in mice with epidermis-specific deletion of IKK2. Nature 417, 861-866 (2002).

48. Reiley, W., Zhang, M. \& Sun, S.-C. Tumor suppressor negatively regulates JNK signaling pathway downstream of TNFR members. J. Biol. Chem. 279, 55161-55167 (2004)

49. Reiley, W. W. et al. Deubiquitinating enzyme CYLD negatively regulates the ubiquitin-dependent kinase Tak1 and prevents abnormal $\mathrm{T}$ cell responses. J. Exp. Med. 204, 1475-1485 (2007).

50. Cvijic, M. E., Xiao, G. \& Sun, S. C. Study of T-cell signaling by somatic cell mutagenesis and complementation cloning. J. Immunol. Methods 278, 293-304 (2003).

51. Uhlik, M. et al. NF-kappaB-inducing kinase and IkappaB kinase participate in human T-cell leukemia virus I Tax-mediated NF-kappaB activation. J. Biol. Chem. 273, 21132-21136 (1998).

\section{Acknowledgements}

We thank M. Pasparakis and J.M. Penninger for mutant mice, X. Lin for cell line, B.E. Gilbert for influenza virus and R. Lin, K. Ye and C. Wang for expression vectors. We also thank the personnel from the NIH/NCI-supported resources (flow cytometry, DNA analysis, histology and animal facilities) under award number P30CA016672 at The MD Anderson Cancer Center. This study was supported by grants from the National Institutes of Health (AI057555, AI064639, GM84459 and AI104519) and partially supported 
by a pilot fund from the Center for Inflammation and Cancer at the MD Anderson Cancer Center.

\section{Author contributions}

J.Y. and X.Z. designed and conducted the research, prepared the figures and wrote a part of the manuscript; M.C., M.N., J.-H.C. and Y.X. contributed to the experiments; T.Z. and A.Z. contributed to the T-cell imaging experiment, S.D.-E. and W.C. contributed to the PBMC experiment; J.W.L. contributed multiple sclerosis patient PBMC samples; and S-C.S. supervised the work and wrote the manuscript.

\section{Additional information}

Supplementary Information accompanies this paper at http://www.nature.com/ naturecommunications
Competing financial interests: The authors declare no competing financial interests.

Reprints and permission information is available online at http://npg.nature.com/ reprintsandpermissions/

How to cite this article: Yu, J. et al. Regulation of T-cell migration by the kinase TBK1 during neuroinflammation. Nat. Commun. 6:6074 doi: 10.1038/ncomms7074 (2015)

\section{(c) (1)}

This work is licensed under a Creative Commons Attribution 4.0 International License. The images or other third party material in this article are included in the article's Creative Commons license, unless indicated otherwise in the credit line; if the material is not included under the Creative Commons license, users will need to obtain permission from the license holder to reproduce the material. To view a copy of this license, visit http://creativecommons.org/licenses/by/4.0/ 